\title{
Effect of Different Physical Training Forms on Change of Direction Ability: a Systematic Review and Meta-analysis
}

\author{
Hallvard Nygaard Falch, Håvard Guldteig Rædergård and Roland van den Tillaar * (B)
}

\begin{abstract}
Background: The ability to perform a rapid change of direction (COD) is a critical skill in numerous court- and fieldbased sports. The aim of this review is to investigate the effect of different physical training forms on COD performance.

Methods: A systematic review of the literature was undertaken using the following databases: PubMed, SPORTDiscus and Google Scholar. Studies were eligible if they met the following criteria: (1) a COD test measuring performance before and after the training intervention, with specific description of the test in terms of length and number of changes in a direction with specified angles, (2) involve training intervention like plyometric, strength, sprint, specific COD training, or a combination of these training forms targeting the lower extremities, (3) the study had to state training background in terms of which sport they participated in and their competitive level and a detailed methodological description. Non-English articles were excluded. Percentage difference and effect sizes were calculated in order to compare the effects of different training interventions.
\end{abstract}

Results: A range of studies performing plyometrics, strength, sprint, specific COD training, training with postactivation potentiation or a combination of these training forms were examined. The percentage of change and effect size (ES) were calculated. Seventy-four studies met the inclusion criteria, comprising 132 experimental groups and 1652 unique subjects. The review revealed no clear consensus on which training form is optimal to develop COD performance. All training forms resulted in an increase in performance from almost no ES to large ES.

Conclusions: The results of the study indicate that COD ability is a specific skill, whereas the COD task, the sports require determines which training form is the most effective to develop COD ability. Training targeting improvement in COD performance should address the duration of the training in line with which energy system is utilized. The complexity of the COD task with respect to the individual athlete must be considered. Consequently, the number of changes in direction and the angles of the task are relevant when organizing training.

Keywords: COD, Effect size, Training form, Specificity

\section{Key Points}

- This meta-analysis highlights the effect of different physical training forms on change of direction ability based in court- and field-based sports.

- In general, strength training is sufficient to develop strength-oriented COD, plyometric training is effective in developing both strength- and velocity-

* Correspondence: roland.v.tillaar@nord.no

Department of Sport Sciences and Physical Education, Nord University, Odins veg 23, 7603 Levanger, Norway

(c) The Author(s). 2019 Open Access This article is distributed under the terms of the Creative Commons Attribution 4.0 International License (http://creativecommons.org/licenses/by/4.0/), which permits unrestricted use, distribution, and reproduction in any medium, provided you give appropriate credit to the original author(s) and the source, provide a link to the Creative Commons license, and indicate if changes were made. oriented COD, and sprint training is beneficial for velocity-oriented COD.

- Factors such as biological age, experience, type of sport and test assessment also have a large influence on what training form should be performed to enhance COD ability in court- and field-based sports

\section{Background}

In court- and field-based sports, there are different requirements to gain a higher level of performance. An \\ SpringerOpen}


athlete must possess a reasonable level of different skills and capacities. Furthermore, position on the field, playing style and the specific demands of the sport may influence the factors an athlete must cope with [1]. Movement speed is unarguably a central component of many sports. Movement speed can be differentiated into acceleration, maximum speed and agility [2]. The emphasis on movement speed and being able to move quickly in a new direction can provide a physical and tactical advantage over the opponent and is particularly important in sports such as soccer, handball, basketball and rugby [3].

In court- and field-based sports, most of the game is performed at low intensity $[4,5]$. Despite the low intensity work, a substantial amount of work is done at high intensity, otherwise known as maximal action, and the ability to repeat maximal actions can be of great relevance $[4,5]$. The ability to execute maximal actions in key moments can decide the outcome of a match, such as scoring or preventing a goal [6]. Sprinting, tackling and rapid changes in direction are typical examples of maximal actions and contribute significantly to total energy expenditure [7].

Research has shown that soccer athletes accomplish approximately 700 direction changes during a game of varying intensity, and 600 of these changes in direction are 0 $90^{\circ}$ turns [8]. Roughly 50 of the direction changes in a soccer match are performed at maximal intensity [9].

Data from Póvoas et al. [10] reveal that handball players use a great amount of force during tasks that require changes in direction. Despite the fact that most games are completed at low intensity, the study states that stops, deceleration and changes in direction represent $60 \%$ of the physical actions executed in a game. In basketball, 20\% of the sprints involve rapid changes in direction [11], which is fewer than the aforementioned sports [8, 9]. Furthermore, data from Duthie et al. [12] suggest that rugby is the sport with the fewest rapid changes in direction compared to the other studies mentioned in this review. Their study revealed that only $16 \%$ of all the sprints in rugby included a rapid change in direction. In court- and field-based sports like football, handball, rugby and basketball, it is suggested that the ability to perform rapid changes in direction is an important factor in relation to match outcomes [13]. Due to the specific demands in different sports, pre-planned situations will occur where the ability to perform a rapid change in direction is limited to the athlete's physical capacity. Therefore, the ability to perform a rapid change in direction is determined by the athlete's ability to produce a high amount of force in a relatively short time [3].

\section{Change of Direction}

Rapid change of direction (COD) includes an acceleration phase and a deceleration phase, followed by acceleration in a different direction [14]. The acceleration phase is similar to the acceleration in a sprint, which is characterized by lower vertical displacement of centre of mass $(\mathrm{COM})$, which in turn enables greater horizontal ground reaction force to be exerted. The manoeuvre responsible for a COD features eccentric muscle work during braking, followed by concentric muscle work, granting propulsive force [15]. A rapid COD in response to a stimulus has been defined as agility $[13,14,16,17]$. Without a stimulus, agility is limited only by physical determinants and is therefore termed COD [3]. There are several physical determinants that could influence COD performance, as suggested by Young et al. [17].

\section{Physical Determinants of COD}

Different physical determinants affecting COD performance are anthropometrical dispositions and technical qualities, straight sprinting speed, and strength qualities in lower extremities [17] (Fig. 1).

\section{Anthropometrics}

Change of direction performance relies on greater acceleration of body mass and therefore a lower percentage of fat mass, along with great relative strength are desirable [18]. The mechanical forces, expressed via Newton's laws of motion can explain this. Newton's first law of motion states that an object (athlete) remains at a constant velocity unless acted upon by a force. This means an athlete must apply force to the ground in order to shift in speed. The necessary force required for a change in speed and direction is dependent on the athlete's body mass, on velocity when approaching the COD step, and on the angle of direction change [13].

The influence of body mass upon COD is highlighted by Chaouachi et al. [19] who revealed a high correlation between fat-percentage and performance in COD $(r=$ 0.8 ). Athletes with lower percentage of fat mass completed a COD task in less time. A decrease in fat mass or increase in maximal strength without an increase in bodymass increases the athlete's relative strength, which is beneficial when changing momentum and accelerating in a new direction [13]. Additionally, when changing momentum in a COD task, the athlete is required to rapidly lower the COM for appropriate force production, which is an advantage for shorter athletes, since they commonly possess lower COM than taller athletes. Thereby, they outperform their taller counterparts with significant less completion time in COD tasks [20].

\section{Technique}

Research has shown no clear consensus regarding optimal technique and COD performance. It is assumed that athletes can develop optimal technique through specific 


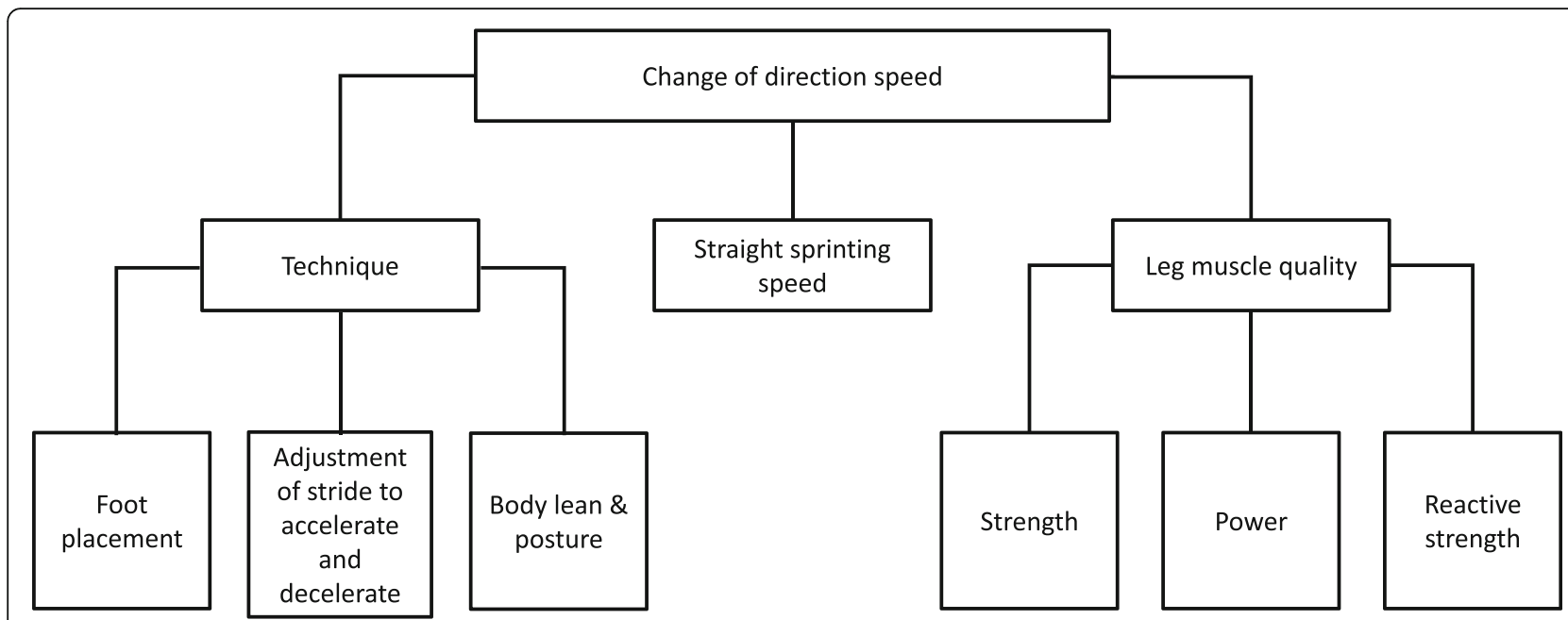

Fig. 1 Flow chart illustrating physical determinants when executing rapid CODs. The figure is modified from Young et al. [17], with permission

COD training themselves since optimal technique is determined by individual anthropometrics and the unique nature of the respective sport [21]. Specific COD training features movement patterns that mimic those performed in competition in terms of COD entry speed, utilization of energy systems, COD angles and number of direction changes [3]. During the COD acceleration phase, a forward shift in COM as a response to forward leaning is required to produce forces horizontal to the ground. A backward lean is necessary to decelerate, and a sideways lean to produce lateral force in order to change direction. Fast postural adjustments and positioning of limbs in COD are essential to produce force in the desired direction and are expected to be very trainable aspects [21]. Furthermore, adjustments of stride to accelerate and decelerate and arm actions are technical factors to be developed when enhancing COD performance [21].

\section{Reactive Strength, Power and Plyometrics}

Due to the restricted time in COD, it is desirable to exert a great amount of force to the ground in a short time. When training with external loads, the rate of force development becomes of relevance. Depending on the COD test, it takes approximately $0.44-0.72 \mathrm{~s}$ to develop maximal force [3], which means that athletes should focus on exerting maximal force in this timeframe in exercises aimed at developing COD performance. Reactive strength is one of the subcategories affecting COD performance (see Fig. 1). Reactive strength is the ability to change from eccentric to concentric muscle action as quickly as possible in a stretch-shortening cycle (SSC), as exemplified in a countermovement jump and COD [21]. Plyometric training seeks to exert a high amount of force in a short time.
The goal is to increase power output, which is determined by the force and velocity involved in a SSC [22]. Similarities with SSC suggest that plyometric training can facilitate COD performance. Previous studies have revealed moderate to high correlation between different jump exercises and COD tests: $r=0.64$ [15], $r=0.71$ [23], $r=0.7$ [24]. The selection of plyometric exercises can be challenging when training for COD, because the magnitude of distance, speed and direction in COD tasks varies by sport. Previous research has proposed that bilateral and unilateral training in different directions performed at body mass and external load should be targeted when performing plyometric training [13].

\section{Straight-line Sprinting Speed}

Previous research has reported moderate to high correlation between straight-line sprinting speed and different COD tests: $r=0.3-0.66$ [24], $r=0.55-0.9$ [25], $r=0.73$ [26], $r=0.59$ [27]. The correlations will be largely influenced by number of directions, approach speed, angle of direction and total test distance. As suggested by Bourgeois et al. [13], CODs that encompass angles below $90^{\circ}$ are more velocity-oriented in contrast to angles exceeding $90^{\circ}$, which are more force-oriented (see Fig. 2). Force-oriented CODs are characterized by a longer completion time at entry of COD, the importance of force capabilities increasing as the magnitude of the COD angle increases. A minor loss of speed and shorter ground contact time characterizes velocity-oriented COD tasks, due to less braking action and smaller direction changes [13]. Most studies that showed a moderate to high correlation between straight-line sprinting speed and COD [25-27] assessed tests with a minimum distance of $15 \mathrm{~m}$ and angles of new direction below $90^{\circ}$, thereby applying velocity-oriented CODs. 


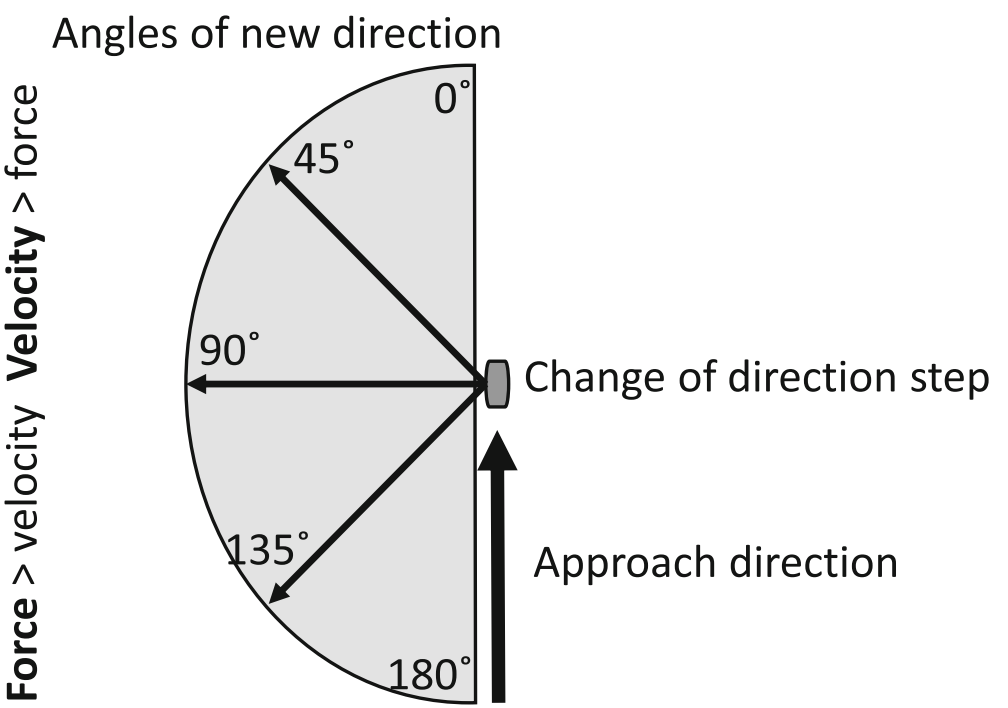

Fig. 2 Graphic illustrating mechanical determinants of COD modified from Bourgeois et al. [13], with permission

A possible explanation for the high correlation values is that the ability to perform fast straight-line sprints is dependent on SSC, while COD and sprints both encompass an acceleration phase. The acceleration phase in COD and straight-line sprint encompass similar technical factors, and an improvement in acceleration ability is likely to improve COD performance over short distances $(0-10 \mathrm{~m})$. This is because an athlete should accelerate quickly after the COD step [13]. However, research argues that sprint and COD are independent skills. Two studies finding low correlation between straight-line sprint speed and COD were Jones et al. [16] $(r=0.50)$ and Hewit et al. [28] $(r=0.39)$. Both studies [16, 28] assessed a COD test with a $180^{\circ}$ angle and a 5-10-m run, which could explain the low correlation between COD and sprint performance, because the COD tests applied in these two studies encompassed strengthoriented CODs [16, 28].

\section{Strength}

In reference to Newton's laws of motion, one can assume that strength capacity is important due to the requirement to overcome inertia in terms of braking and effectively change momentum in a new direction. Increased maximal strength is important if additional strength gain leads to increased relative strength [13]. It would be reasonable to combine bilateral and unilateral strength exercises since deceleration in COD requires great unilateral eccentric strength along with great unilateral concentric strength before and after the change in direction [13, 18]. Markovic [29] revealed low correlation between COD performance and leg extensor strength. However, the COD tests assessed by Markovic [29] were a 20-yard shuttle run and slalom run with several $45^{\circ}$ runs, suggesting the tests were velocity-oriented (Fig. 2), which could explain the low correlation $(r=$ 0.03-0.44).

Studies have found a moderate to high correlation between strength and COD performance $(r=0.4-0.89)$ $[16,30,31]$. The tests assessed in these three studies were a $T$ test or 505 -agility test. What these studies had in common were the angles of new direction, which were $90^{\circ}$ or more, and the short distance for athletes to accelerate. This means that the applied tests were strength-oriented, particularly the 505 test that includes a $180^{\circ}$ turn (Fig. 2).

Chaouachi et al. [19] found moderate correlation between strength exercises and COD performance $(r=$ 0.67-0.69). Their study suggests that eccentric hamstring strength is particularly important when braking during COD tasks. Concentric strength is also important [16], but is a more central aspect when exerting force rapidly during the acceleration phase.

\section{Complex Training}

Complex training involves integration of a strength exercise, typically performed prior to a plyometric exercise. The objective is to increase neuromuscular activity which could increase the performance in a subsequent exercise. It is suggested that greater activation of the fast-twitch muscle fibres occurs, thereby enhancing performance. This is also called the post-activation potentiation (PAP) effect [3]. In Bishop et al. [32], heavy squatting was assessed before performing the proagility COD test (5-10-5 test). They examined optimal resting 
time for COD performance after heavy squatting. The results showed no tendencies towards positive or negative effects. However, not many studies in this area are performed, which makes it hard to draw any conclusions regarding optimal resting time and exercise protocols that may enhance COD performance.

\section{Background and Purpose}

When considering COD as a multifactorial ability, it is suggested that a combination of training addressing multiple factors can lead to greater performance. Improvement of different physical determinants in COD (Fig. 1) can lead to greater COD performance overall [3]. The ability to perform COD effectively is a key aspect in court- and field-based sports [14]. Therefore, the aim of this review is twofold: (1) to investigate how different types of training: strength, plyometric, sprint, specific COD training, training for PAP, or a combination of these approaches can improve COD performance; and (2) to determine the optimal form of training for improvement in COD.

\section{Material and Methods}

\section{Literature Search}

To evaluate the effect of different training interventions upon COD performance, a literature search was completed to structure a meta-analysis. The literature reviewed was attained in August 2019 via the following electronic databases: PubMed, SPORTDiscus and Google Scholar. The following keywords were used in different combinations with change of direction: «Post activation potentiation», «Countermovement jump», «strength», «Plyometric», «Complex», «Training» and «Sprint». Title and abstract were read in order to evaluate the relevance of the articles. During the process of selection of articles, COD tests had to be mentioned in the abstract, with a training intervention targeting performance in lower extremities. The whole paper was read afterwards (Fig. 3)

\section{Inclusion and Exclusion Criteria}

The articles had to contain the following four factors to be included in the study: (1) a COD test measuring performance before and after the training intervention, with specific description of the test in terms of length and

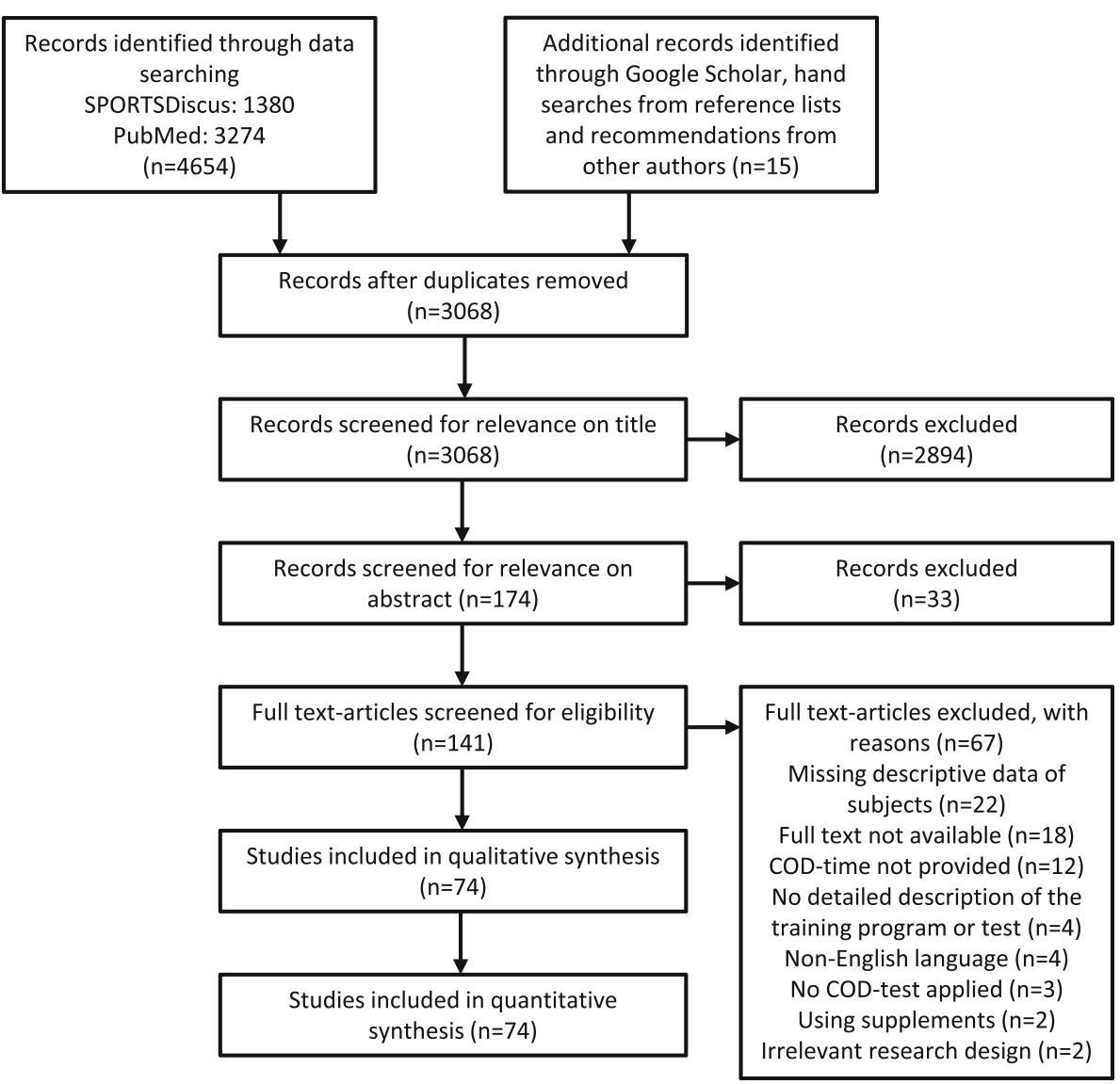

Fig. 3 Search results and identification of studies through the different stages of the systematic review 
number of changes in a direction with specified angles; (2) training intervention performing plyometric training, strength training, sprint training, specific COD training, training for PAP or a combination of these training forms. In addition, workload (volume per training session), number of training sessions per week and number of weeks had to be specified; (3) the study had to state the number of subjects and descriptive statistics concerning individuals' characteristics (height, mass and age), with their training background in terms of which sport they participated in and their competitive level; and (4) a detailed methodological description conducted with reliable measurement tools. The literature search was not limited by sex and age, and there were no restrictions concerning subjects' level of performance/physical conditioning. Articles focused on training that did not target lower extremities were excluded.

\section{Delimitation of Literature}

After processing the literature, 74 articles were included. Many training interventions comprised several experimental groups, meaning that different training categories were sometimes contained within the same article. The training interventions included several groups with differences in training programmes, unequal workload, and differences related to performance or sex. These groups were categorised in tables, making it possible to differentiate the subjects' training improvement based on differences in workload and physical background. A few studies tested the same experimental group in both right and left COD conditions; in these cases, the group will function as two experimental groups.

\section{Design and Structure of Results Tables}

Data from the studies are sorted in Tables 1, 2, 3, and 4, which provide information regarding participants, training interventions, COD tests assessed, improvement in the control group and experimental group from pre- to post-test, and subsequent percentage difference and effect size (ES) in the training intervention. Percentage difference and ES were calculated in order to compare the effects of different training interventions. Effect size was sampled according to Cohen's d $\left(\frac{\mathrm{M} 2-\mathrm{M} 1}{\mathrm{~S}}\right)$. M2 = mean at post-test, $\mathrm{M} 1=$ mean at pre-test. $S=$ pooled standard deviation. ES, from 0.01 to 0.2 were defined as very small, wherein values of $0.2-0.5$ were considered small ES, values of $0.5-0.8$ were considered medium ES, and values of 0.8 or above were considered large ES. Furthermore, ES, 1.2-2 were defined as very large and ES exceeding 2 as huge $[106,107]$

\section{Results}

Subjects

Among the 74 studies attained, there were 132 experimental groups comprising 1652 subjects. Of these, 1146 subjects were soccer players, 69 handball players, 46 tennis players, 25 Australian rules footballers, 56 futsal players, 110 basketball players, 17 volleyball players and 155 players from unknown sports. There was an average of 12.3 subjects for each experimental group with an average age of $16.5 \pm 1.3$. Within the experimental groups, 49 groups assessed plyometric training, eight PAP training, 26 strength training, 20 specific COD training, six sprint training, and 23 groups assessed combined training (Fig. 3). Of the 132 experimental groups, 110 assessed males, seven groups were mixed sex and only 17 experimental groups were comprised of females only. Information regarding each consecutive experimental group can be seen in Tables 1, 2, 3, 4 .

\section{Overview}

The percentage change for the studies varied from a

$2.88 \%$ decrease in performance [85] to a $14.88 \%$ increase of performance [39] (Figs. 4 and 5); ES varied from no ES to huge ES (Figs. 6 and 7). The intervention that displayed the highest change in percentage assessed plyometric training with drop jumps and countermovement jumps [39]. The study displaying the largest ES assessed strength training with squats [63]. Plyometric training resulted in the highest average percentage change overall for experimental groups (Table 1, Fig. 4).

\section{Plyometric Training}

Studies using plyometric training showed an average percentage change from $0 \%[40,60]$ to $14.88 \%$ [39], with no ES to huge ES, respectively (Figs. 4 and 6). The most assessed exercise in the training interventions was countermovement jumps. The study with the highest change in percentage trained in drop jump and countermovement jump [39] (Table 1). Forty-nine experimental groups implementing plyometric training experienced improvement in COD, apart from two groups [40,60]. Twenty-seven groups revealed very small to medium ES [33, 34, 41-45, 47-52, 58, 59, 61, 108]; eleven groups revealed large ES [36, 37, 39, 41, 47-50, 53, 54, 62]; nine groups revealed very large ES [36, 38, 45, 46, 53, 55, 56, 58, 59]; and two groups showed huge ES $[39,57]$. Every experimental group trained twice a week apart from four groups $[40,45,51,53]$. Experimental groups composed of females all shared effects above the medium ES ( $>0.5$ ), except for one study [47]. Only one study [47] assessed a COD test shorter than $10 \mathrm{~m}$. Thirteen experimental groups assessed COD tests under $90^{\circ}$, while 16 groups assessed angles above $90^{\circ}$ and 22 groups performed COD tests above and below $90^{\circ}$. All subjects in the experimental groups were 


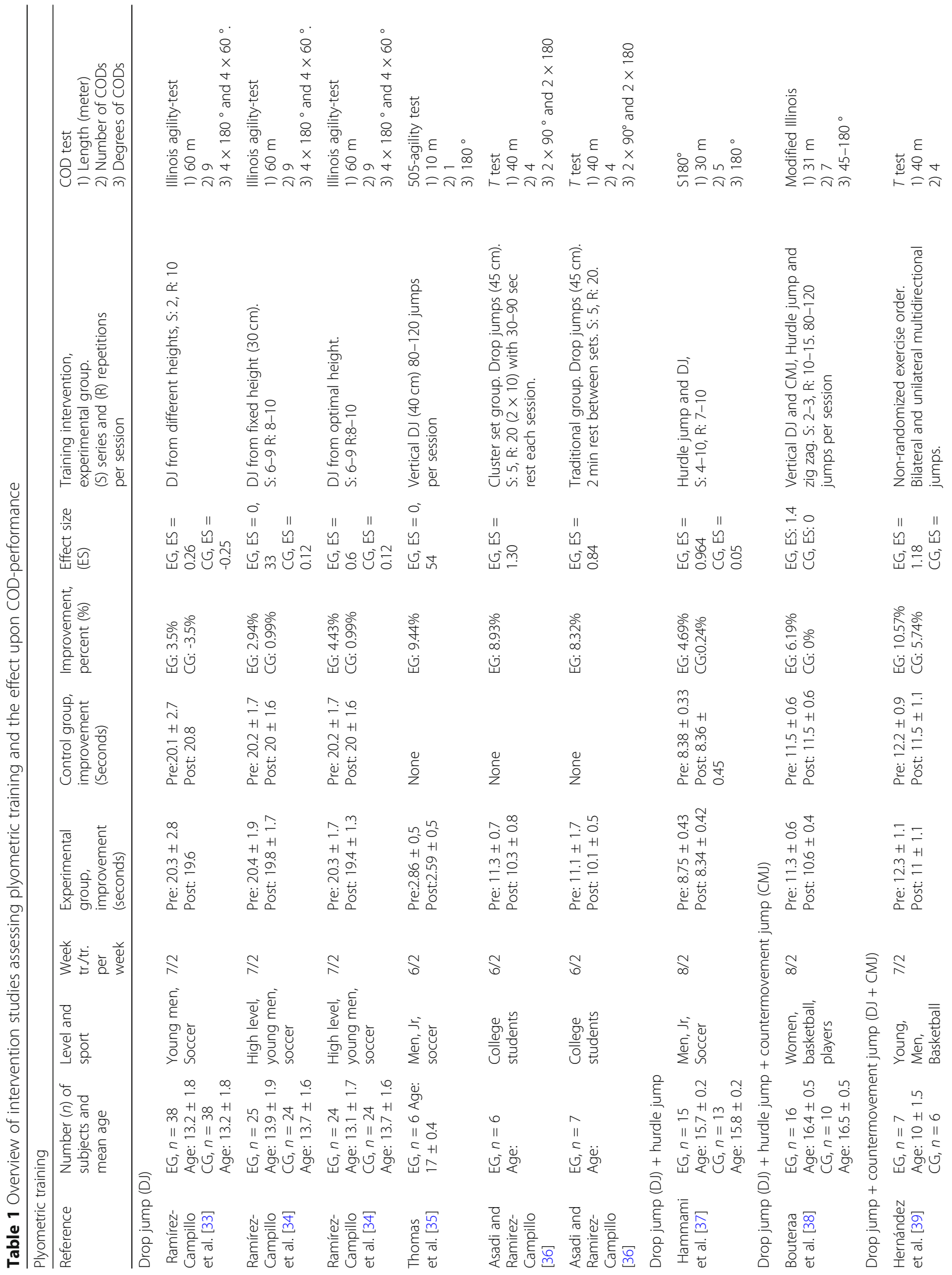




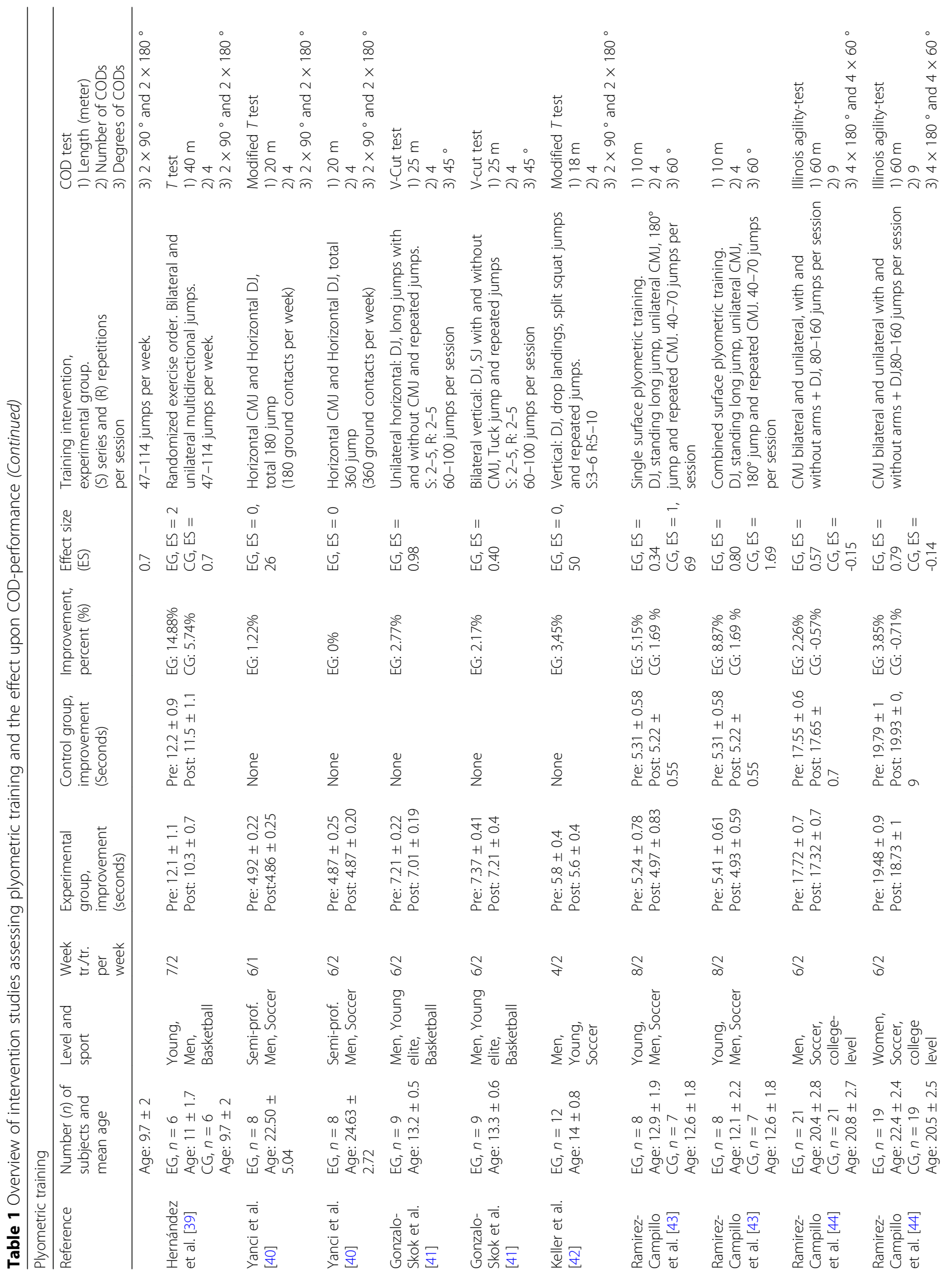




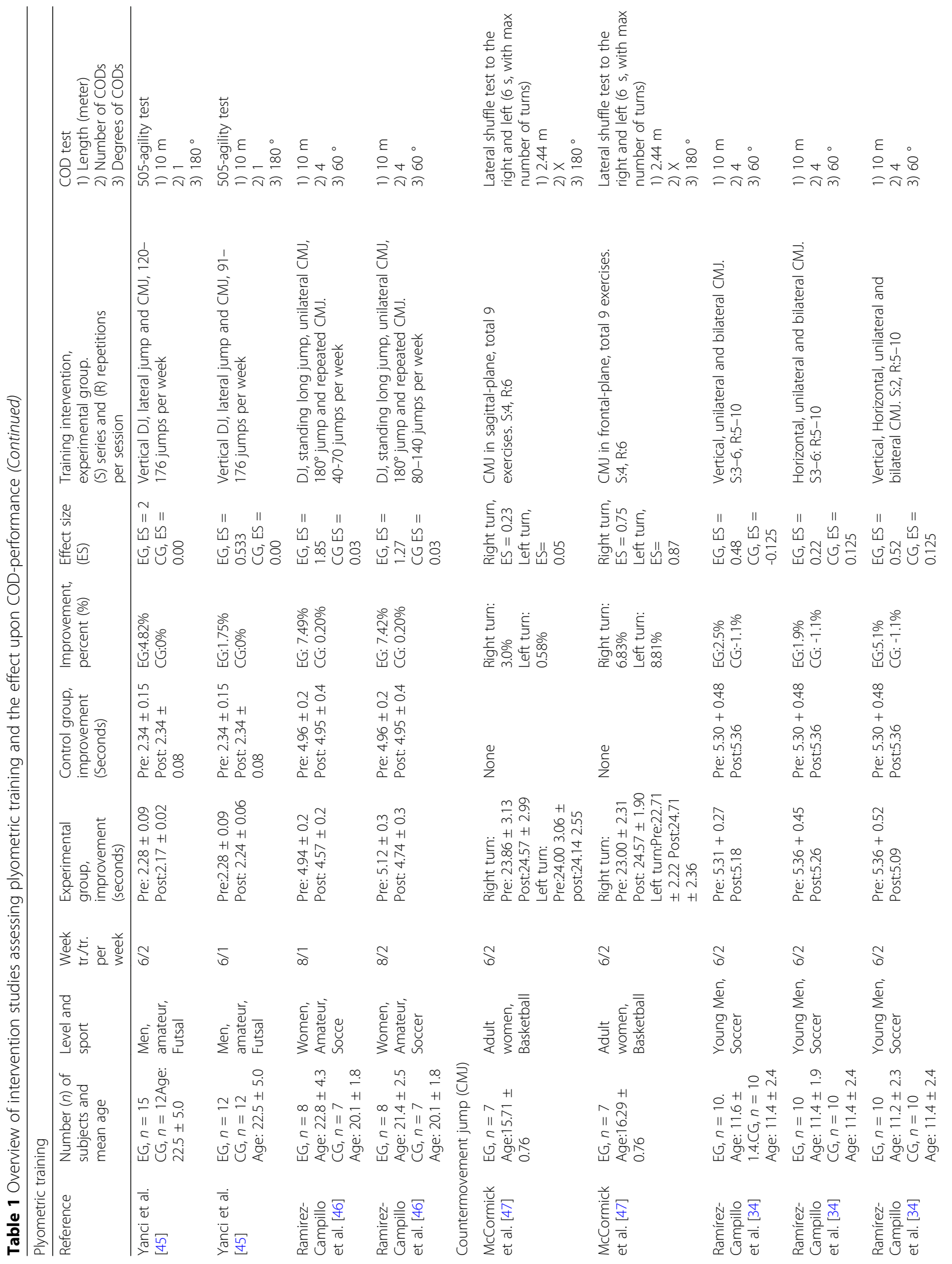




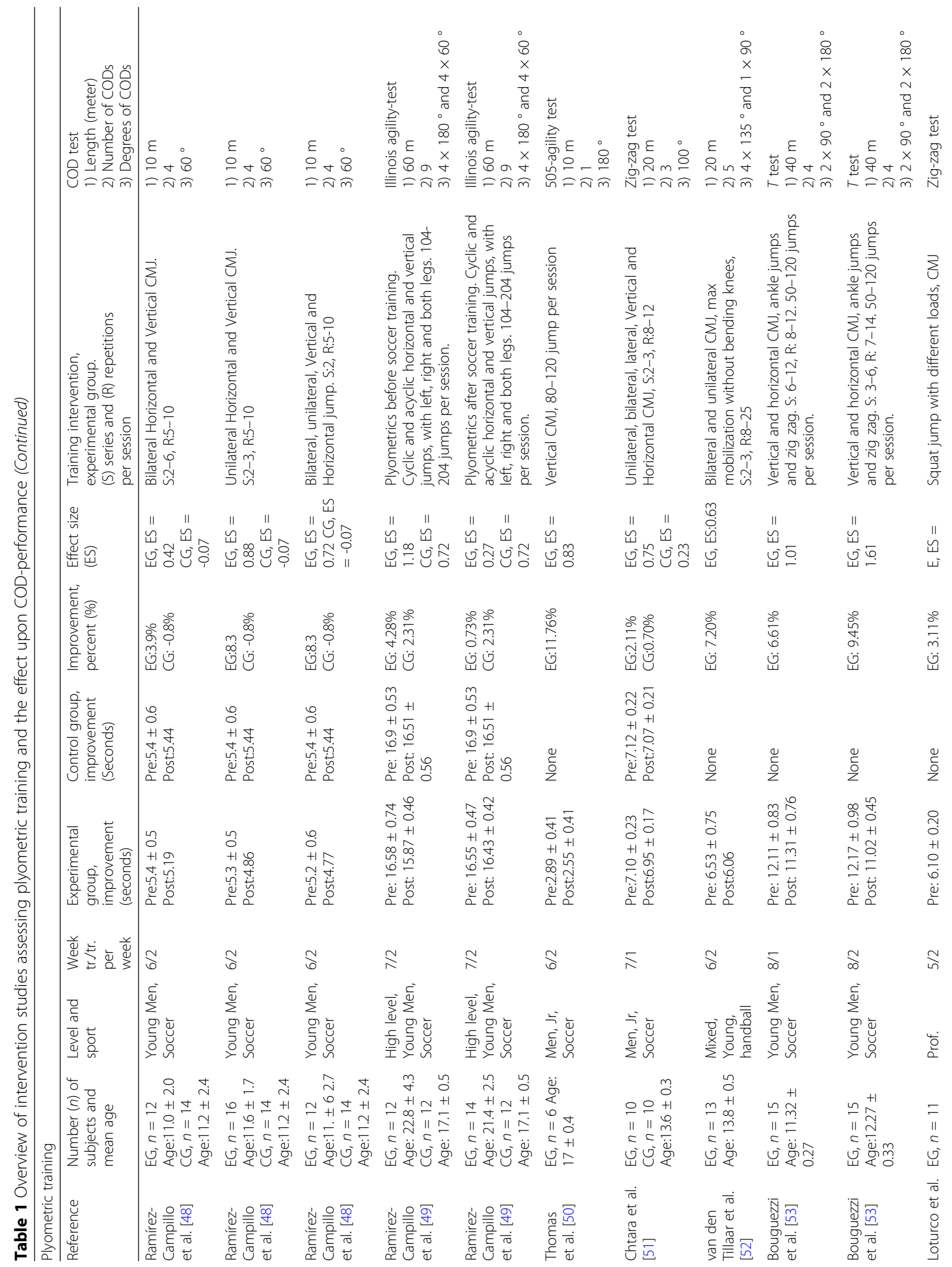




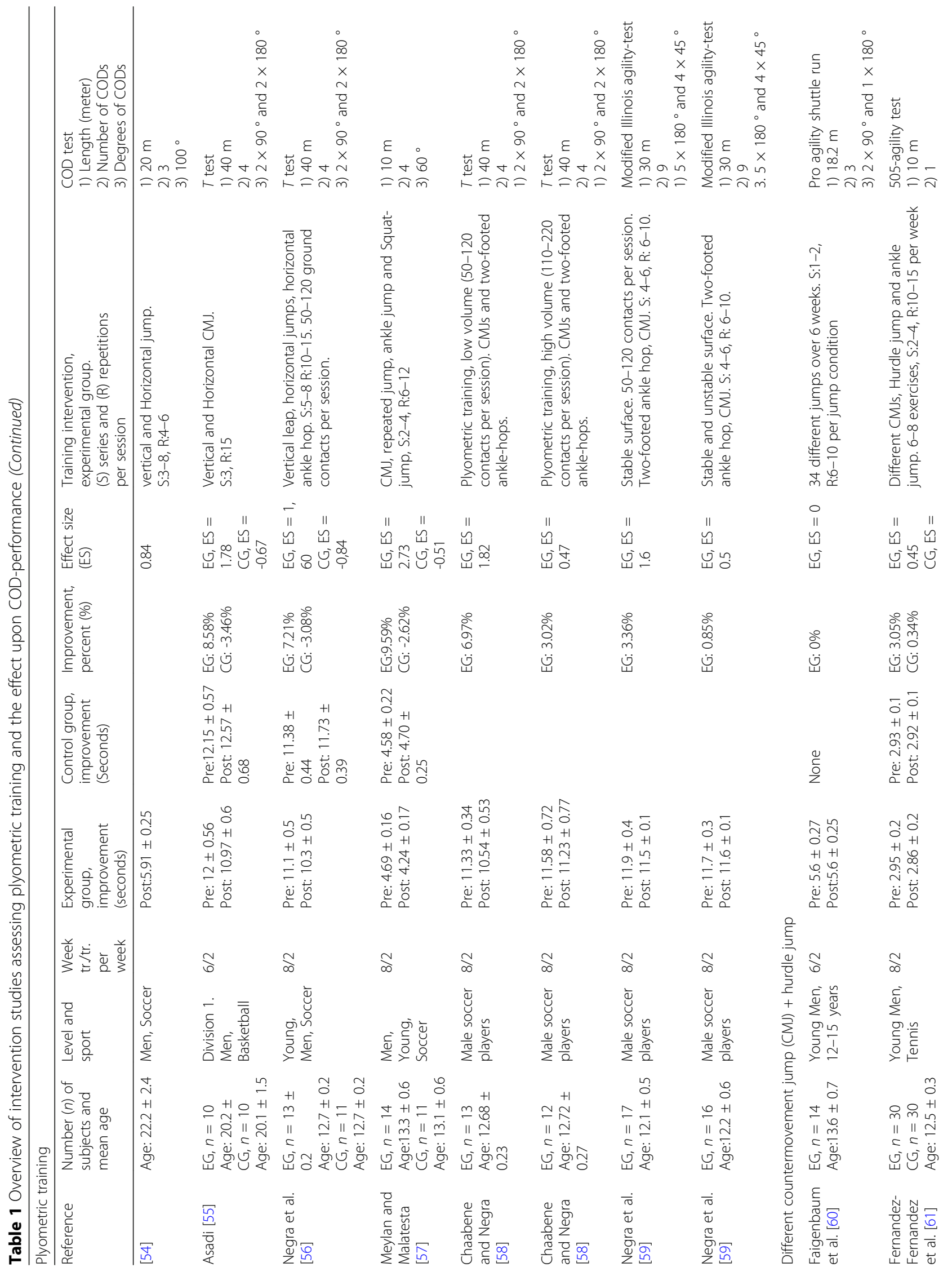




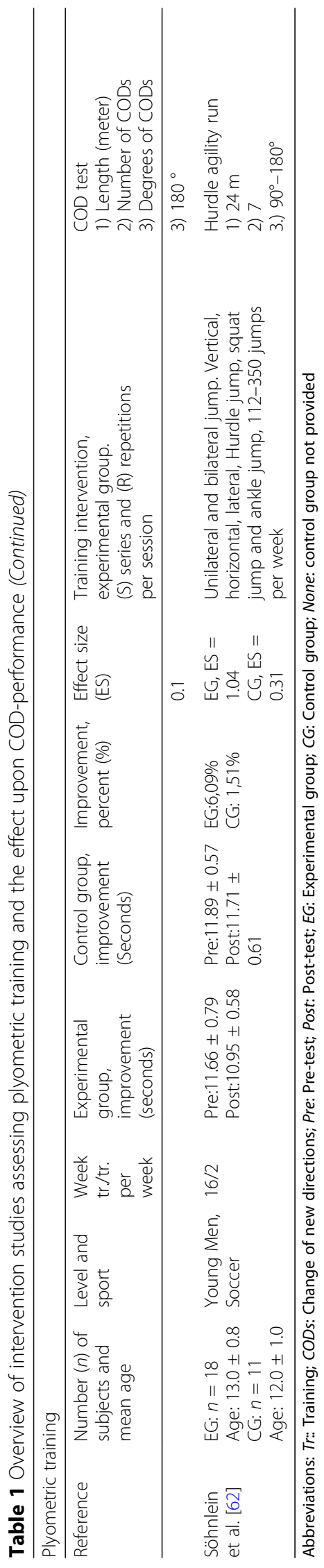




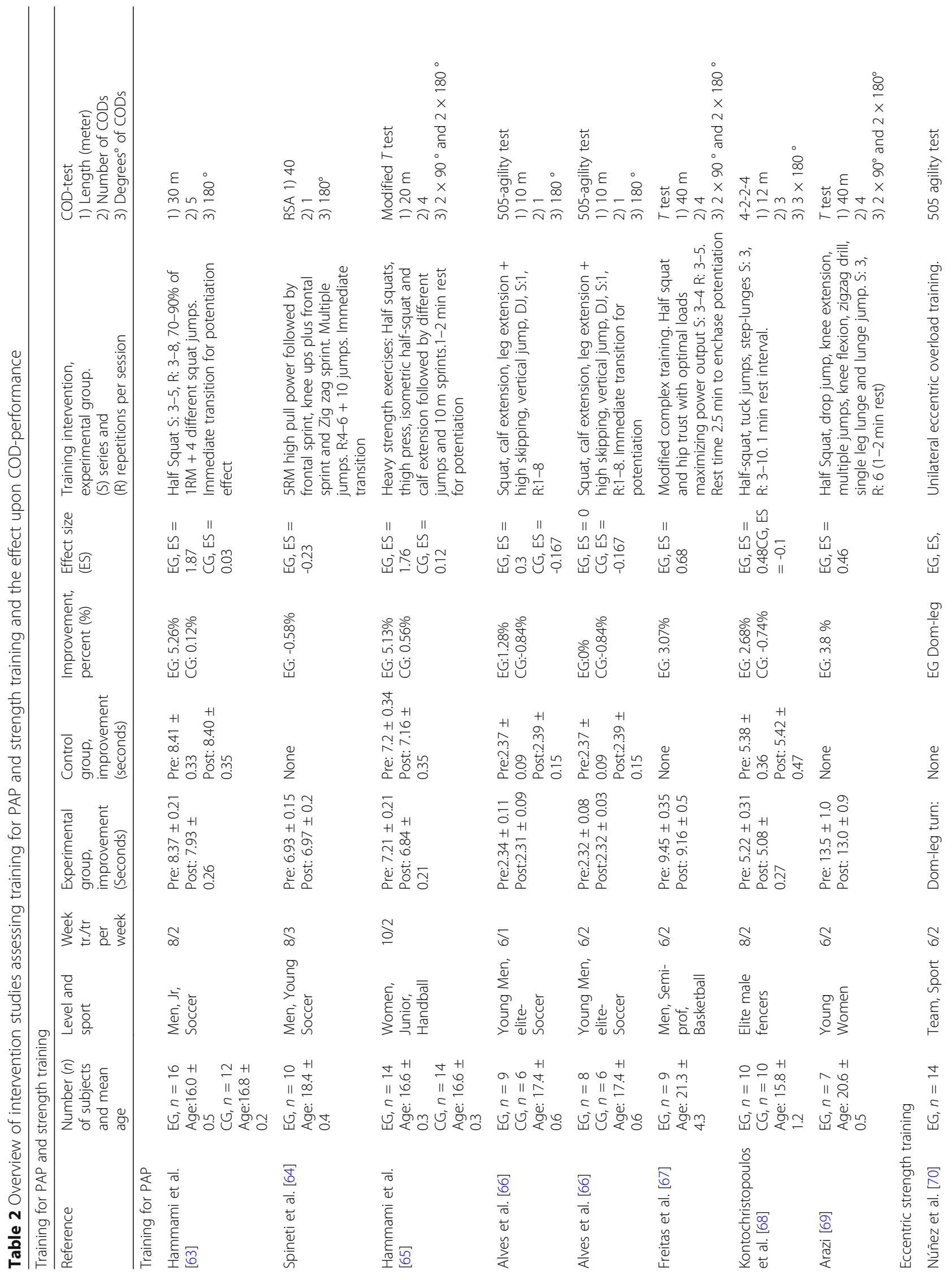




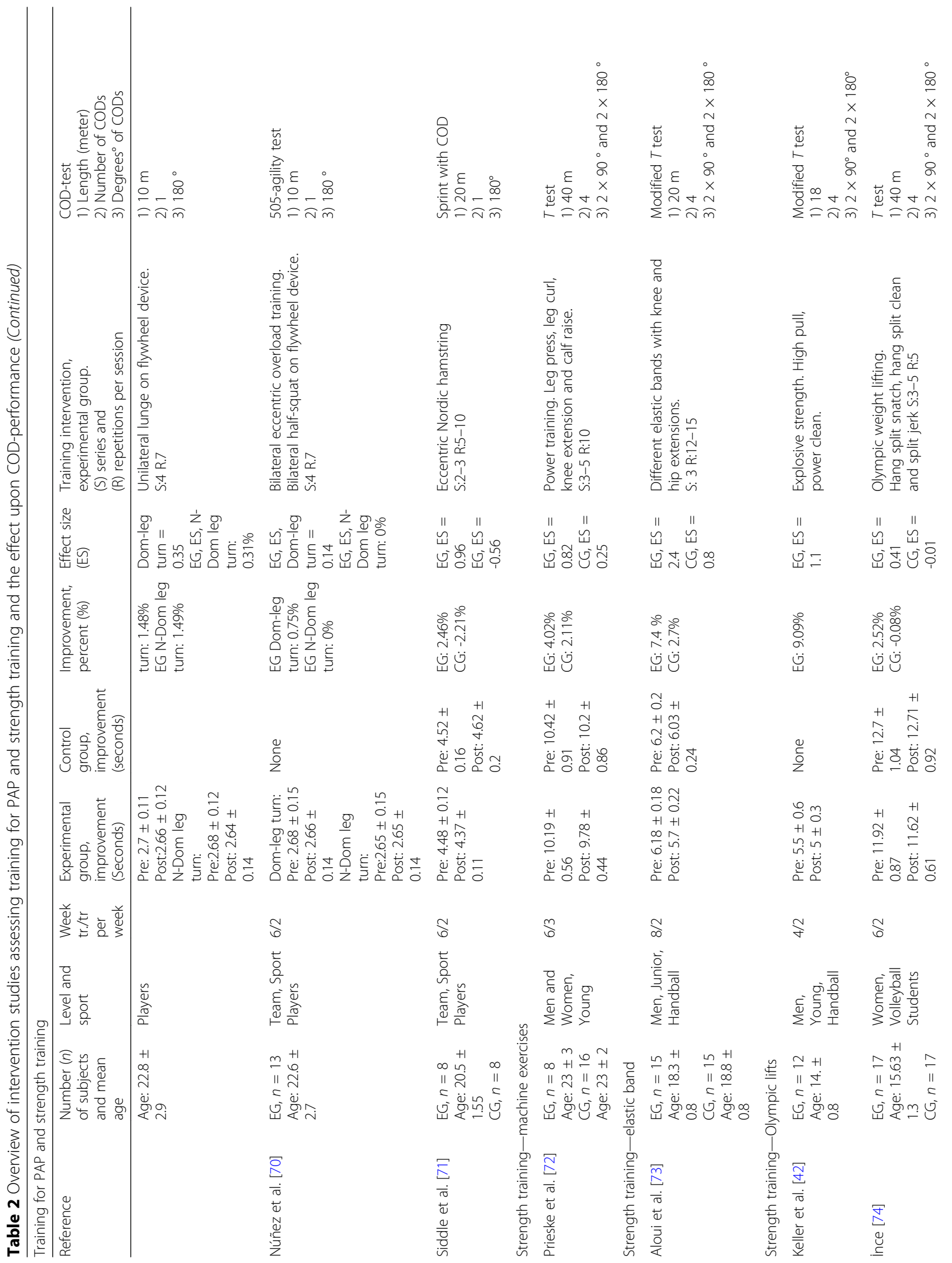




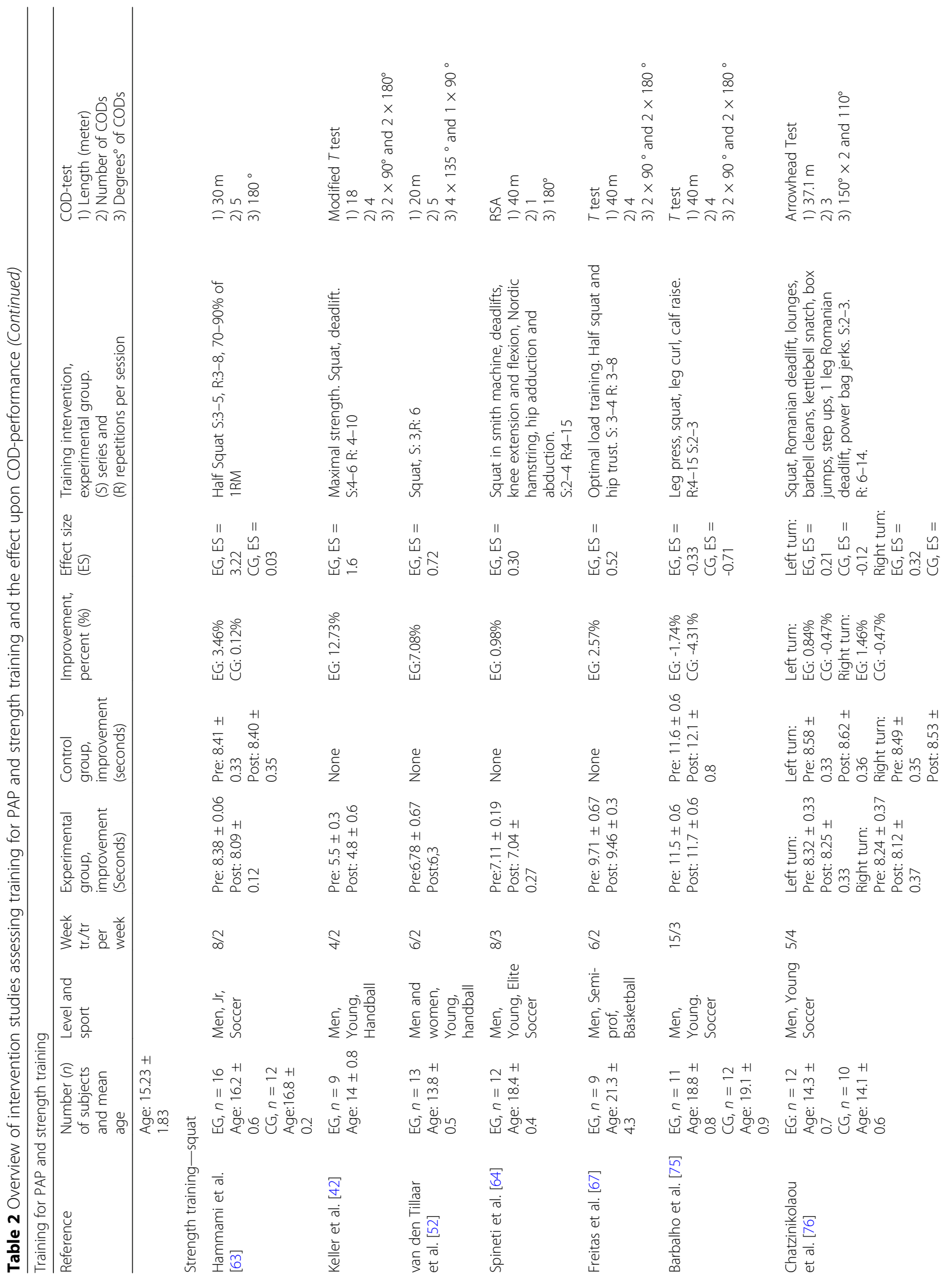




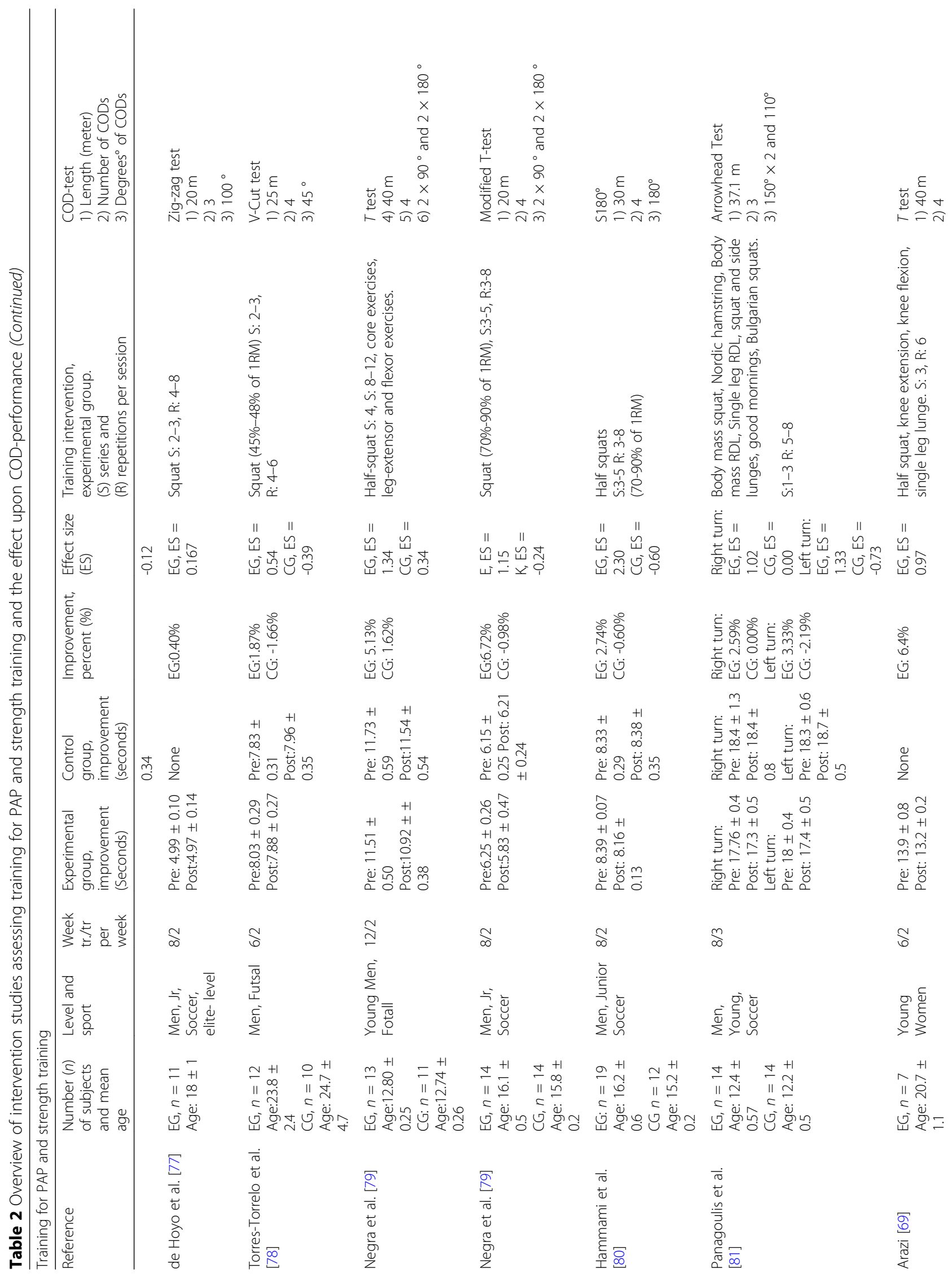




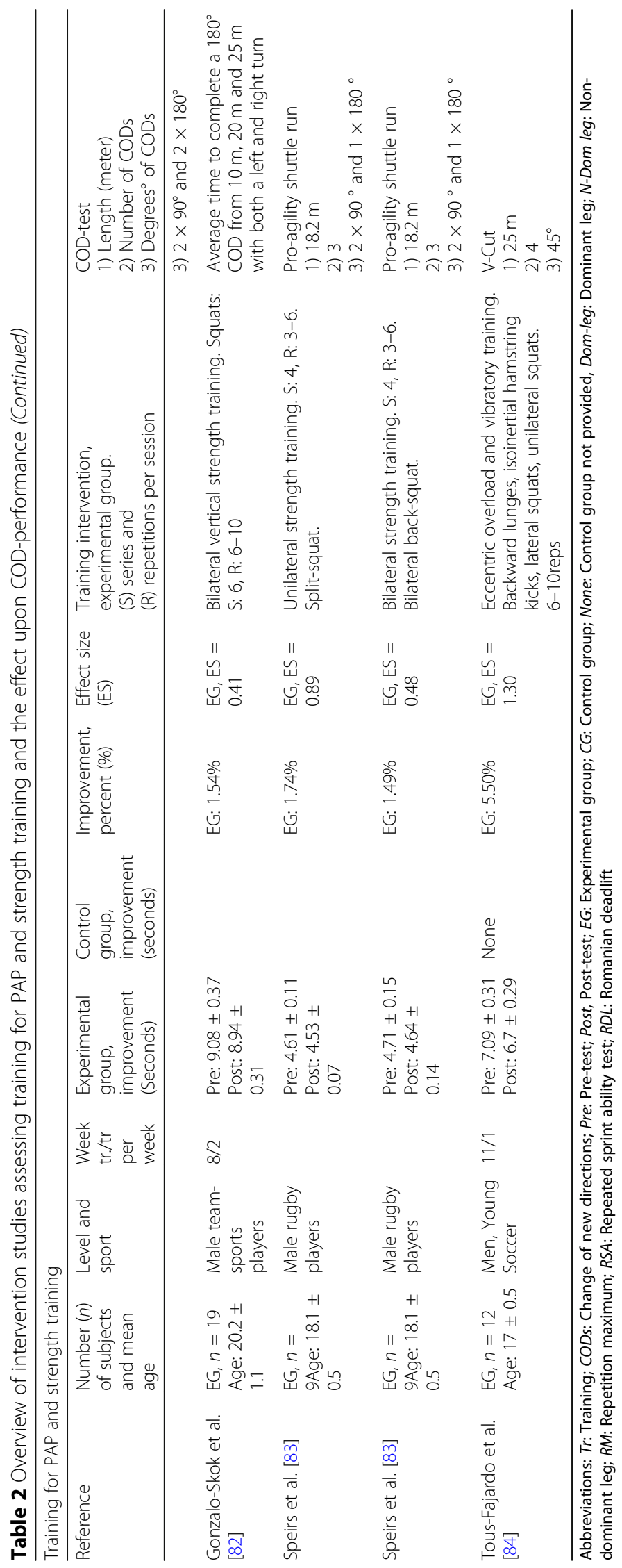




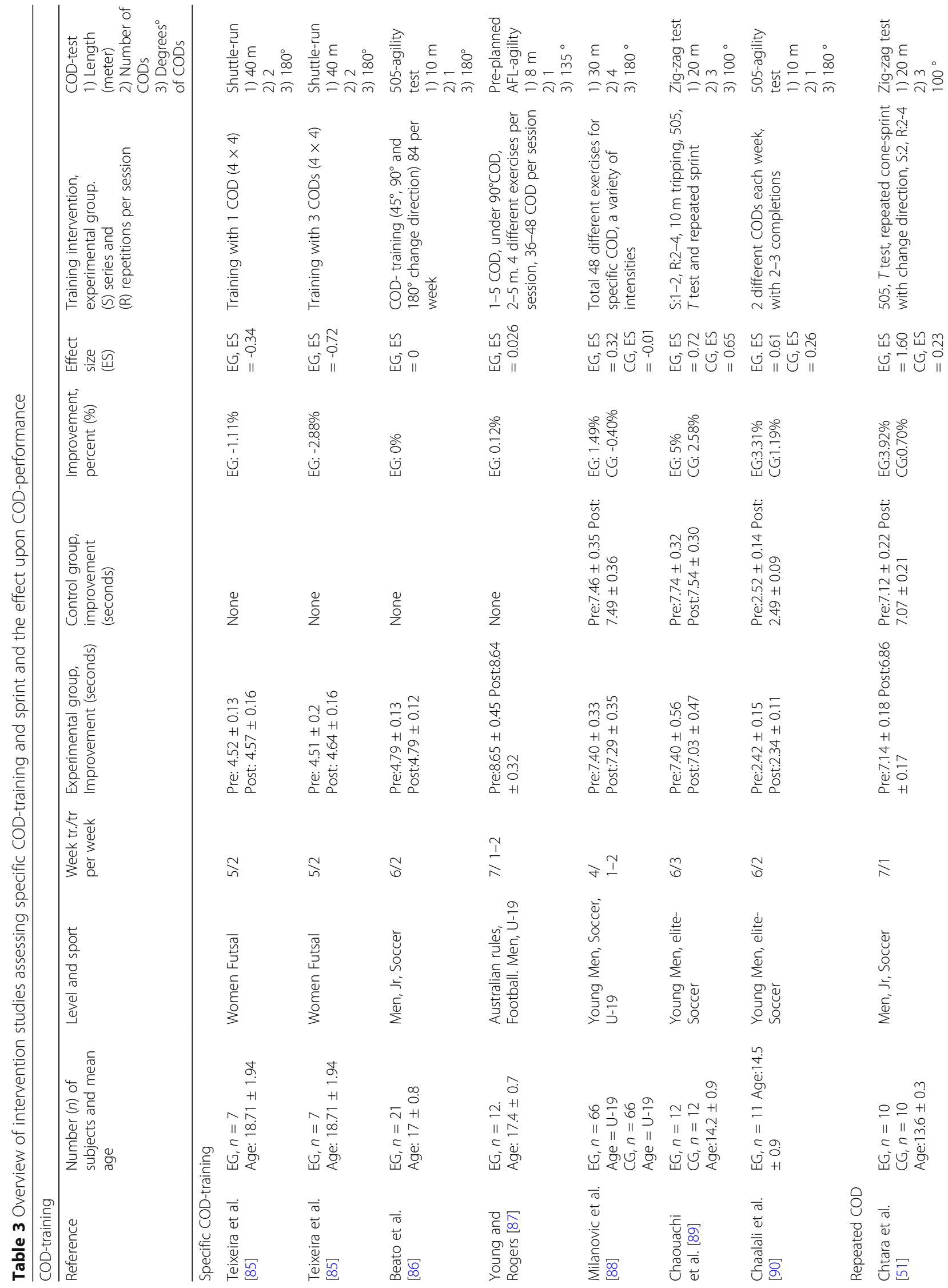




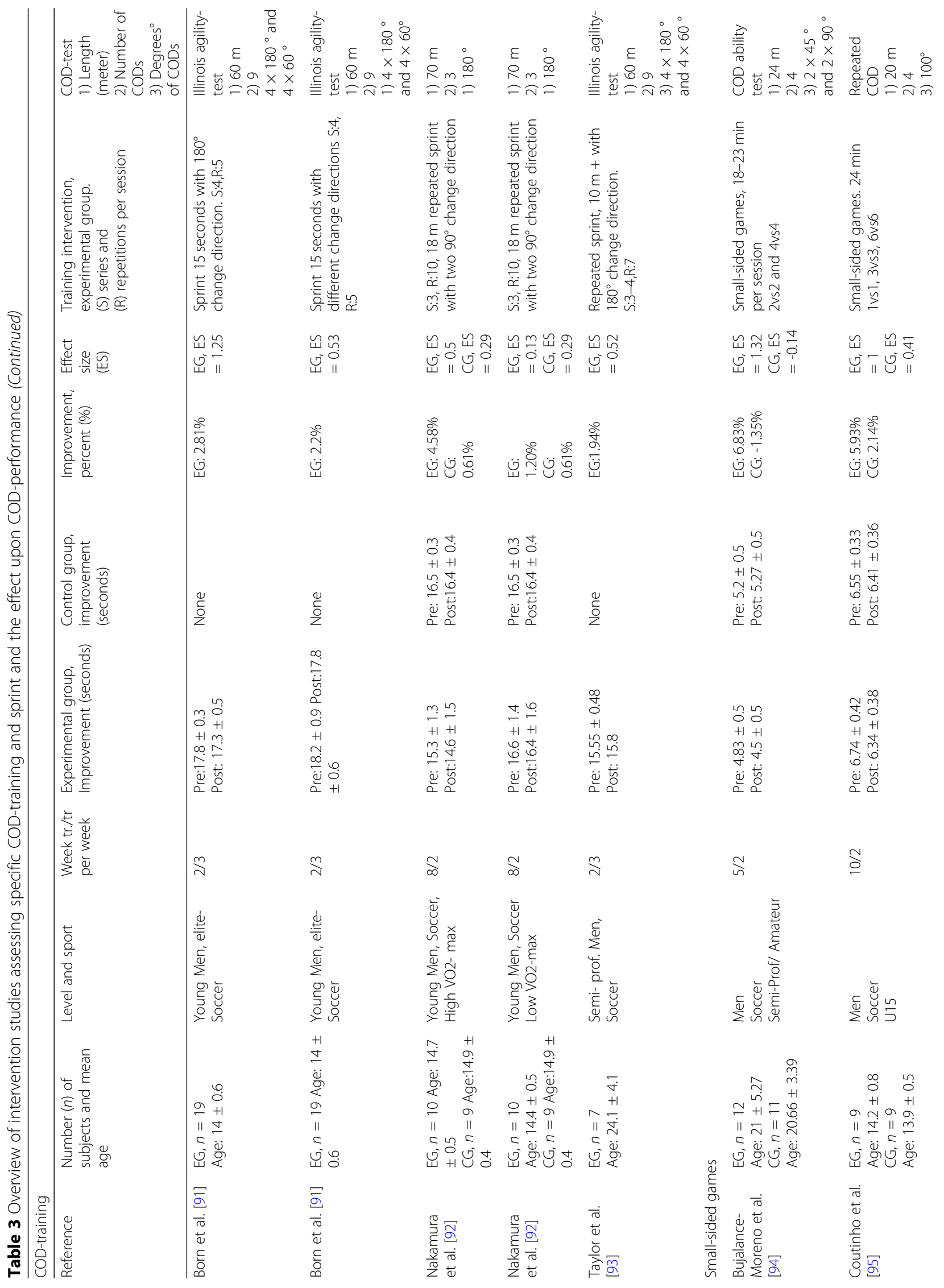




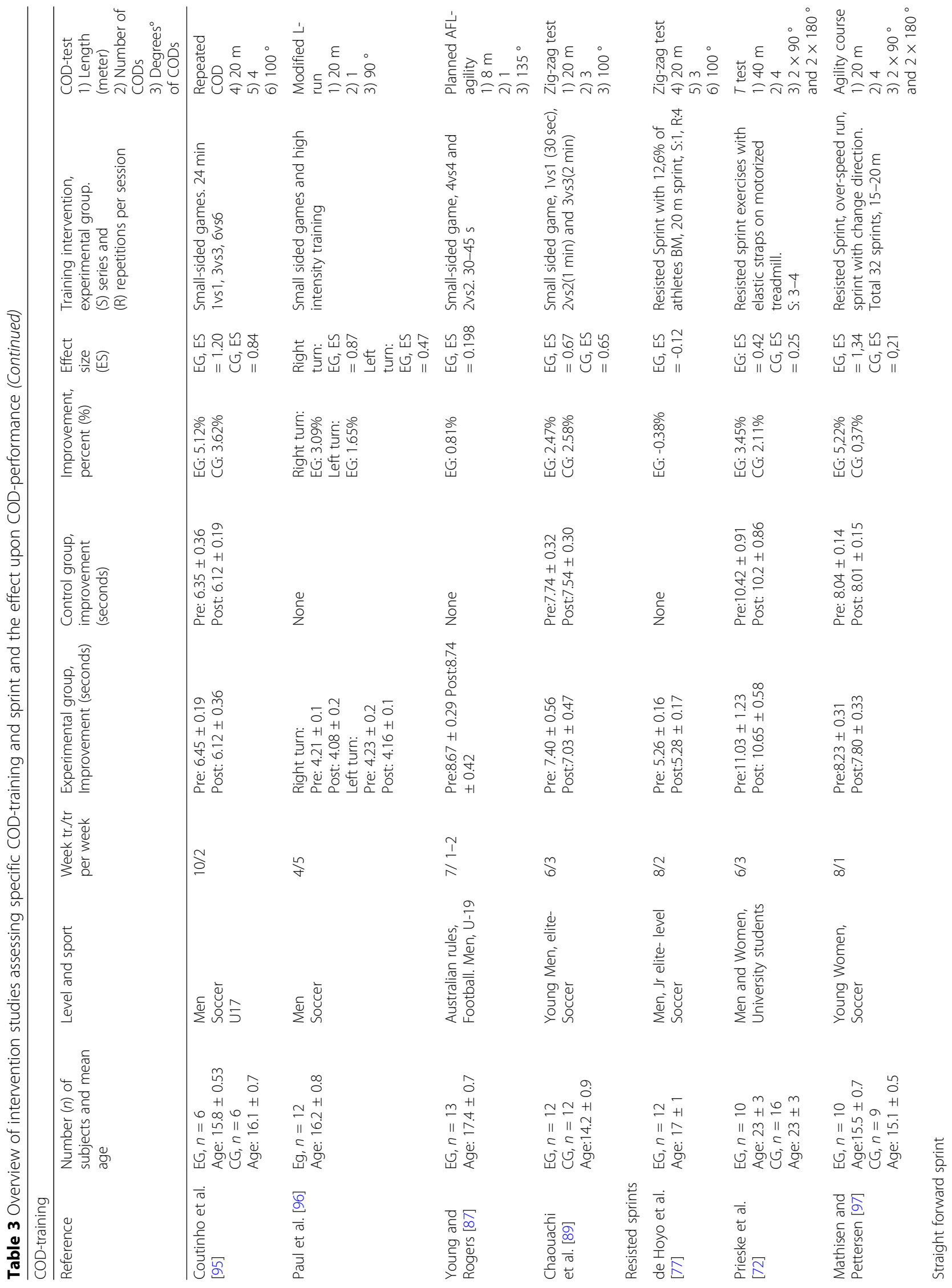




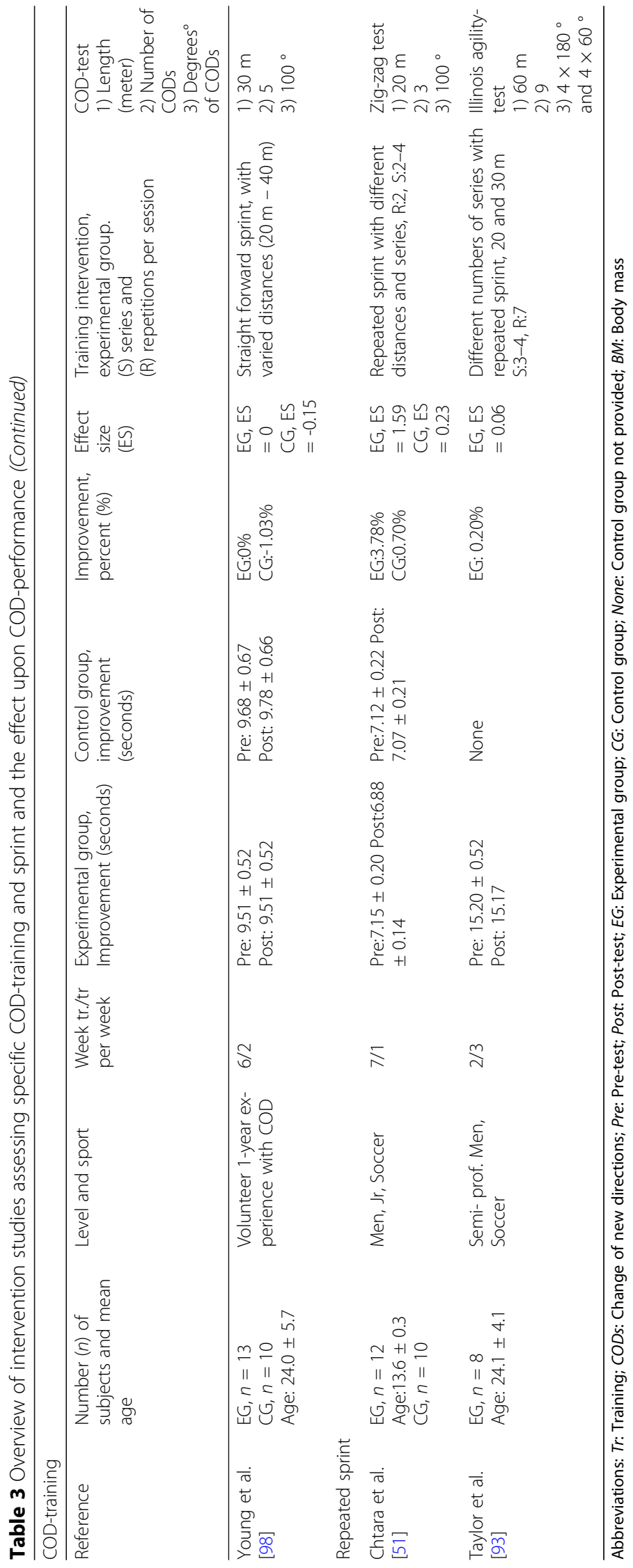




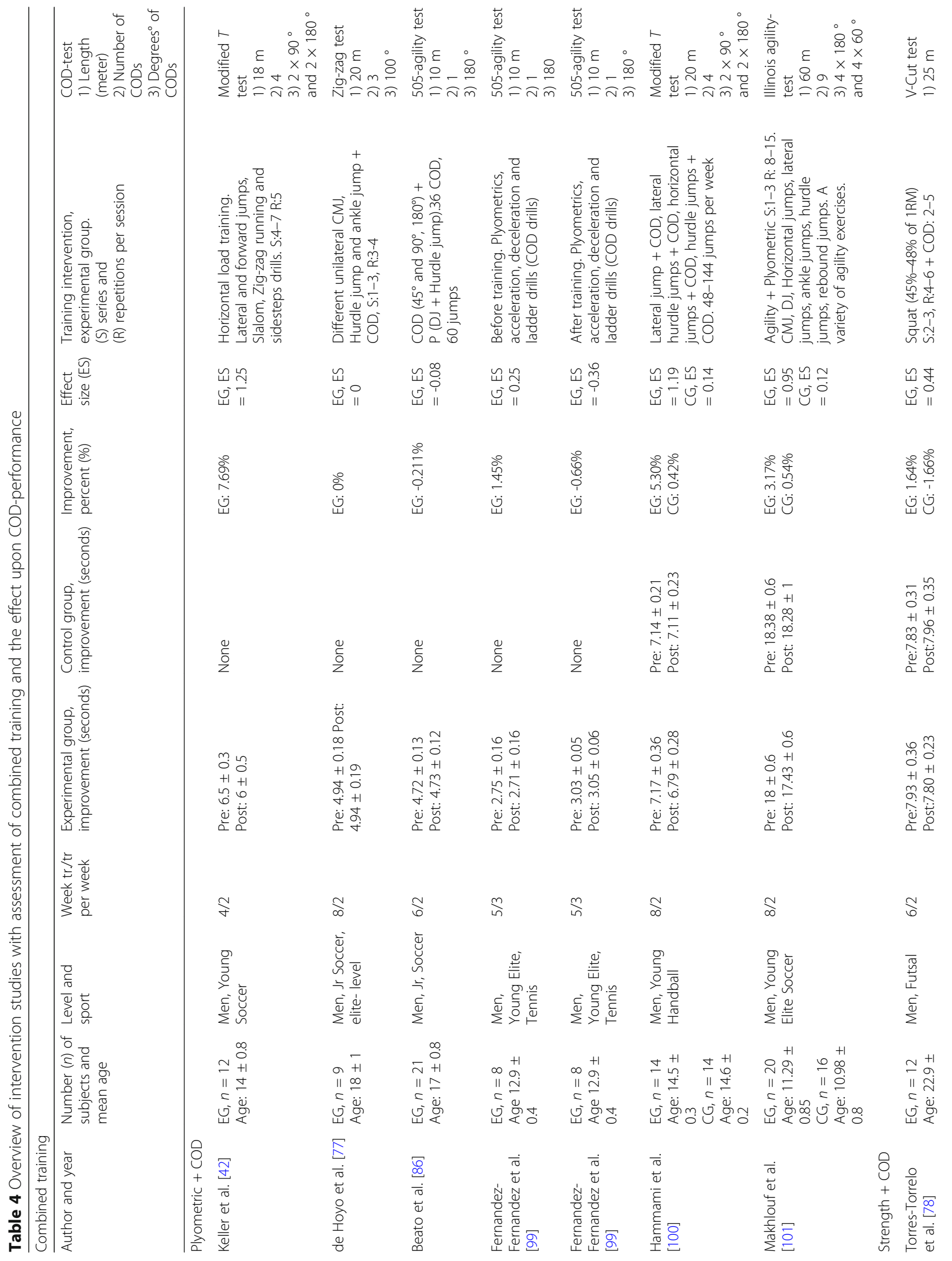




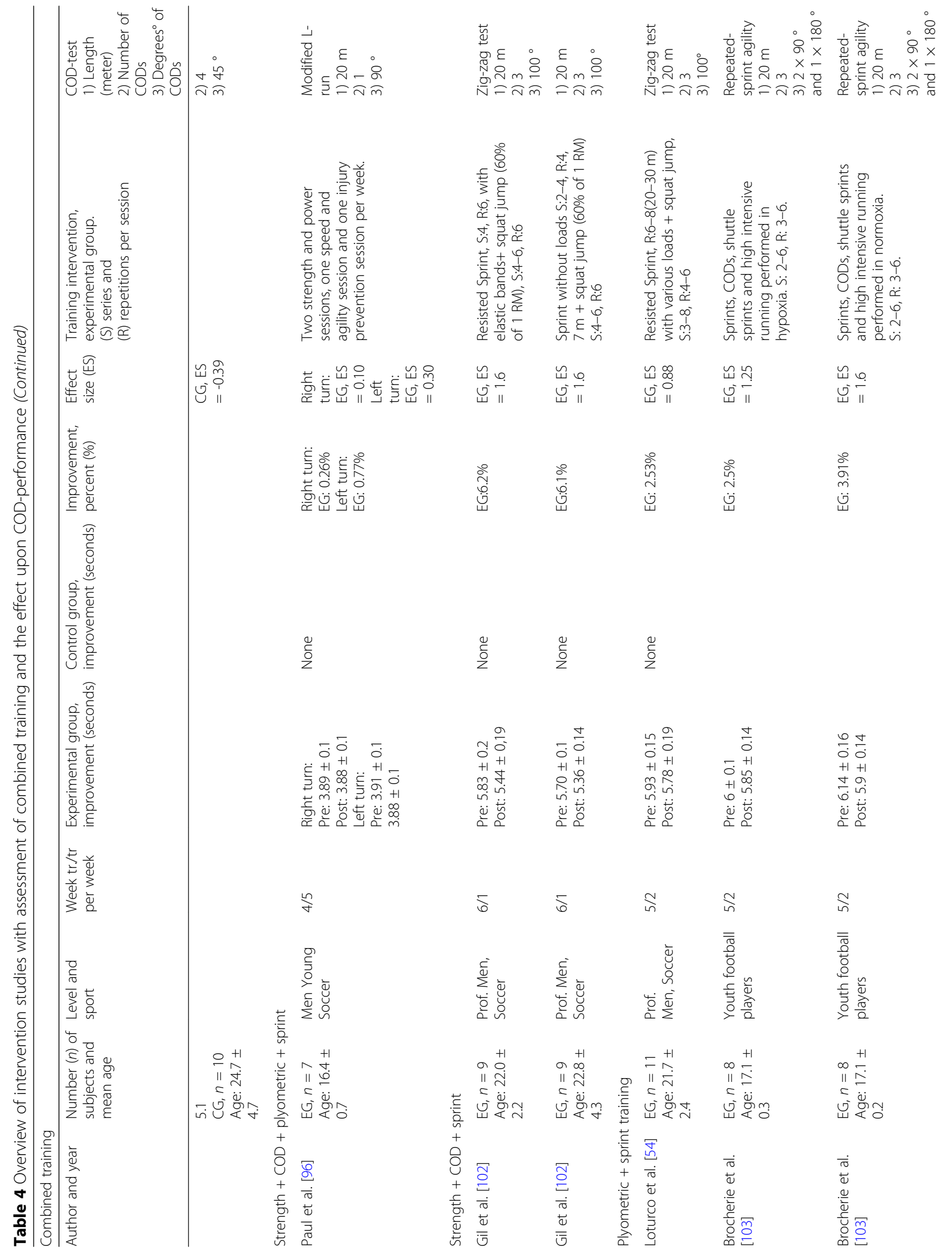




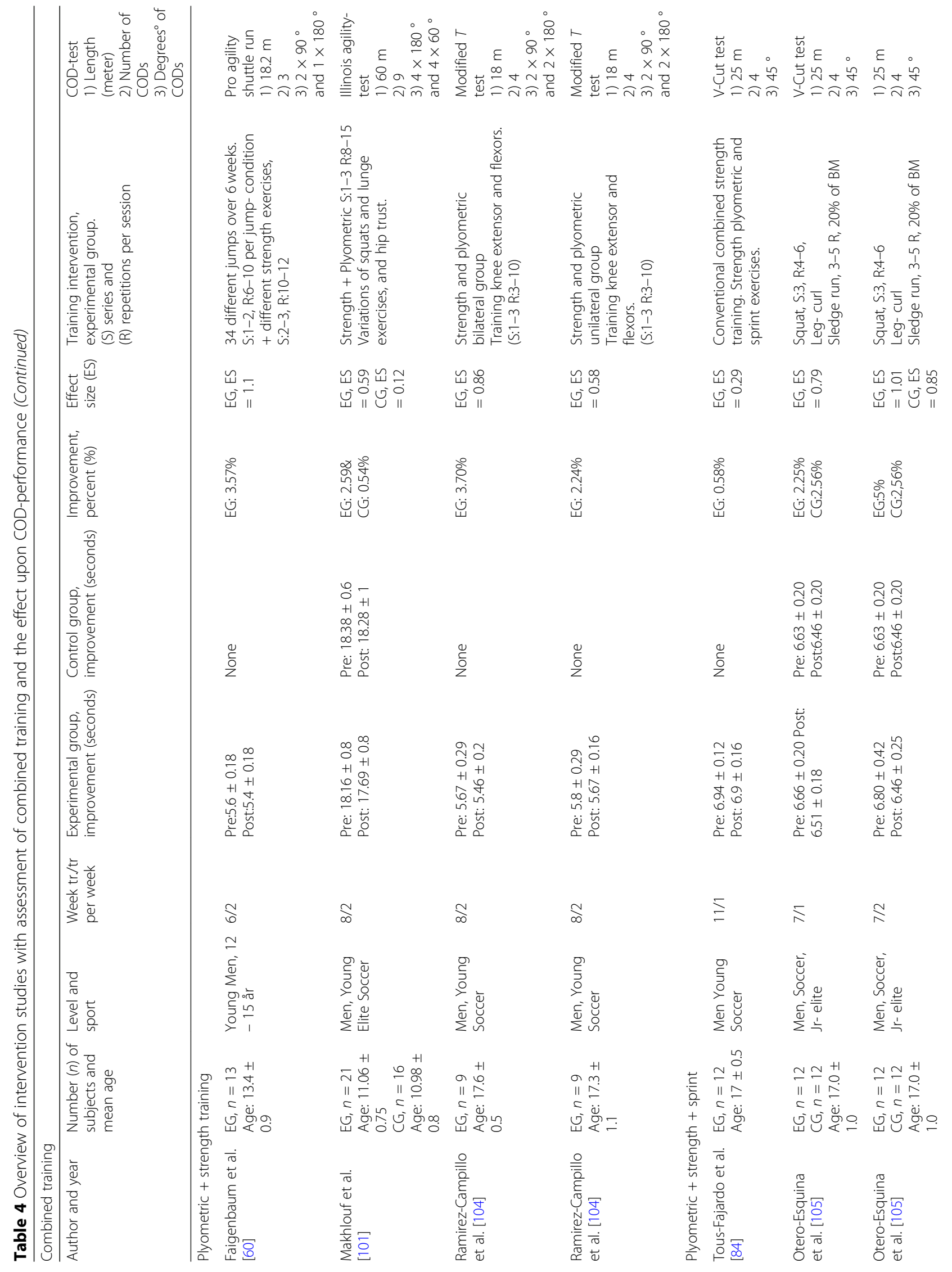




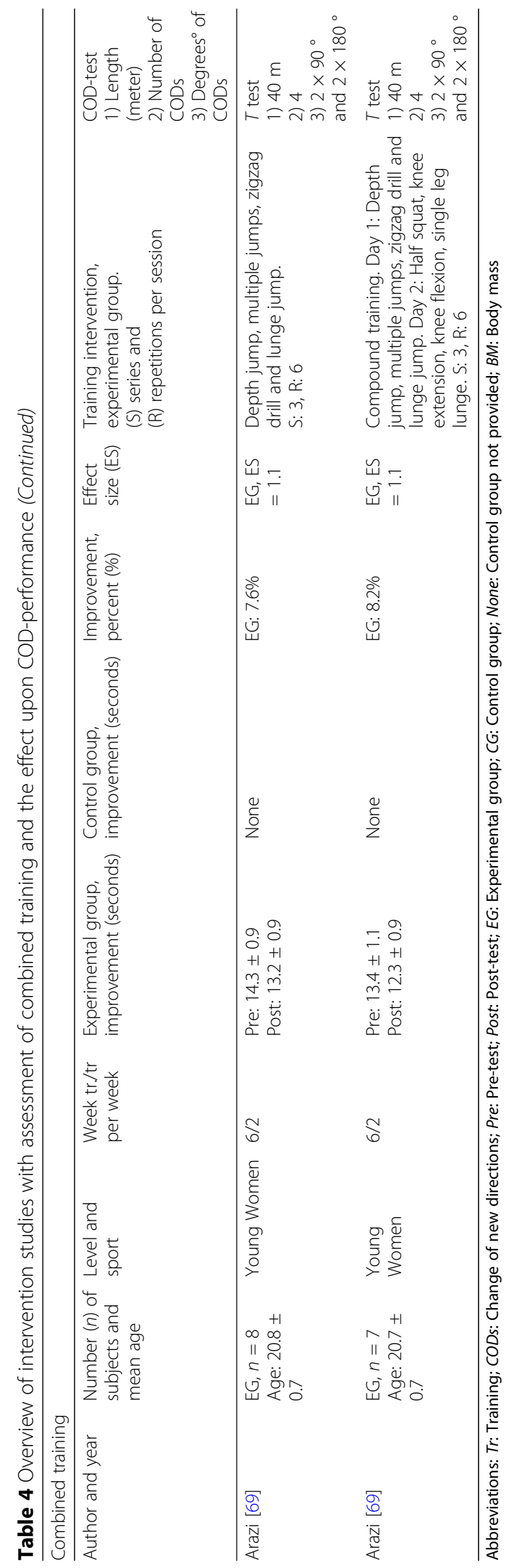




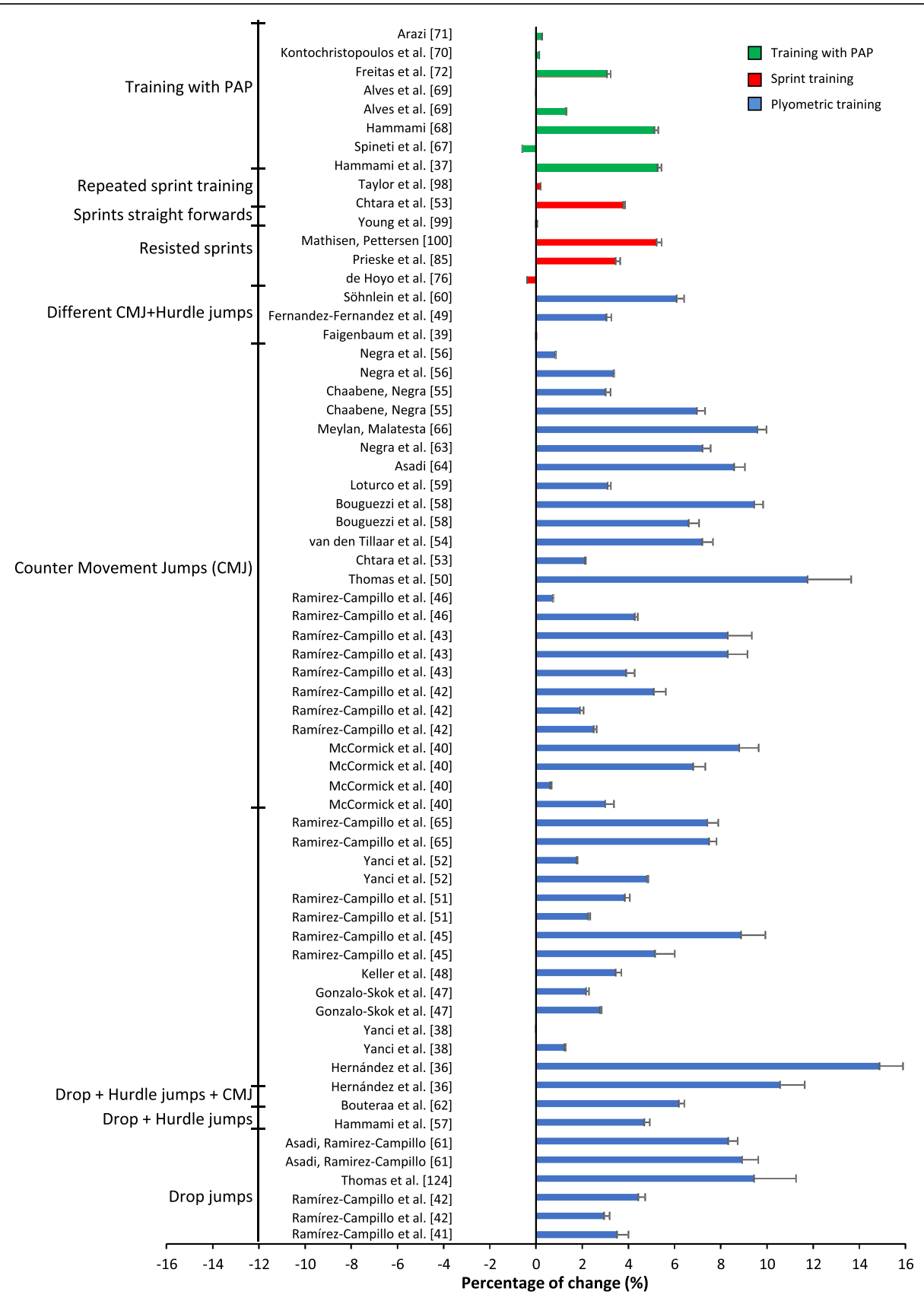

Fig. 4 Percentage of change (+SD) after a plyometrics, sprint or PAP training intervention per training group

young, aged $\approx 24.6$ years [ 40 ] or younger. Plyometric training led to the greatest improvement in overall percentage difference (experimental $5.26 \%$ vs. control $0.98 \%$ ) in comparison to other training forms.

\section{Training for PAP and Strength Training}

Training for PAP resulted in improvement between $-0.58 \%$ [64] and 5.13\% [65] (Fig. 4). Studies assessing PAP trained between one and three times over a period of 6 to
10 weeks. One study exclusively incorporated an exercise that included COD in the training protocol and experienced a decrease in performance [64]. The remaining studies revealed no ES [66], small ES [64, 66, 68, 69] or medium ES [67], followed by two studies revealing very large ES [63, 65] (Fig. 6). The studies revealing very large ES had in common that both experimental groups performed heavy multi-joint exercises followed by different jumps, while one of the experimental groups [65] included an additional 10-m sprint. Training interventions 


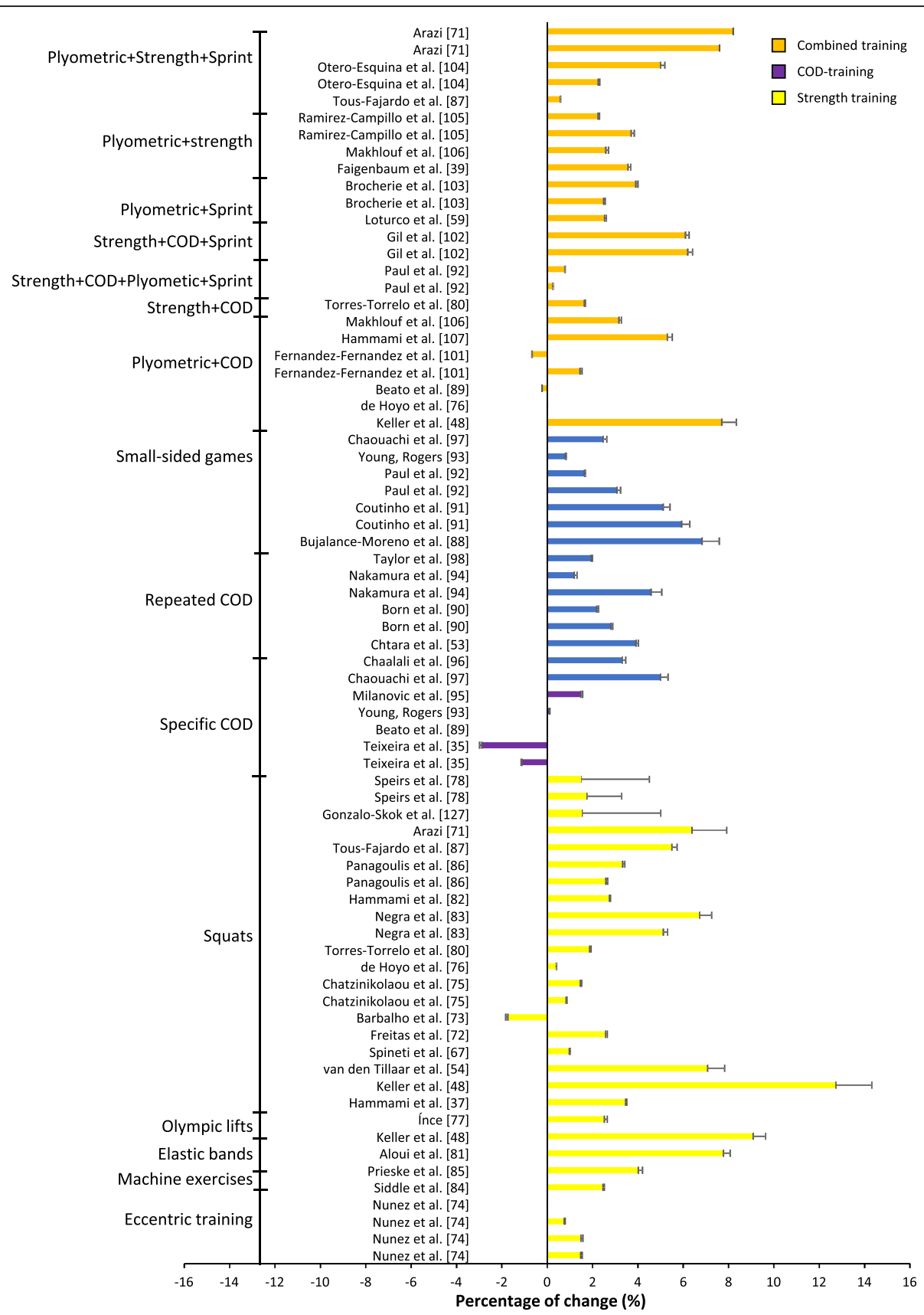

Fig. 5 Percentage of change (+SD) after a combined, strength or COD training intervention per training group

assessing PAP showed a substantial average change in percentage (experimental $2.58 \%$ vs. control $-0.26 \%$ ).

Studies assessing strength training revealed a change in average percentage between $-1.74 \%$ [75] and $12.73 \%$ [42] (Fig. 5). Most studies trained twice a week for 6 to 8 weeks. The study [42] with the largest improvement in percentage trained with half squats (Table 2). Two experimental groups $[70,75]$ experienced no improvement. Twelve experimental groups with an average age of 19 years displayed below medium ES [64, 70, 74-77, 82, 83] and trained 2.5 times a week on average. Three experimental groups with an average age of 19.6 years displayed medium ES [52, 67, 78], training twice a week. Twelve experimental groups comprising 16.5year-old males exceeded large ES [42, 63, 69, 71-73, $79-81,83,84]$ and trained 2.2 times a week on average. All tests applied to measure COD improvement were between 10 and $40 \mathrm{~m}$. Strength training revealed a positive average change in percentage (experimental $3.32 \%$ vs. control $-0.40 \%)$. 


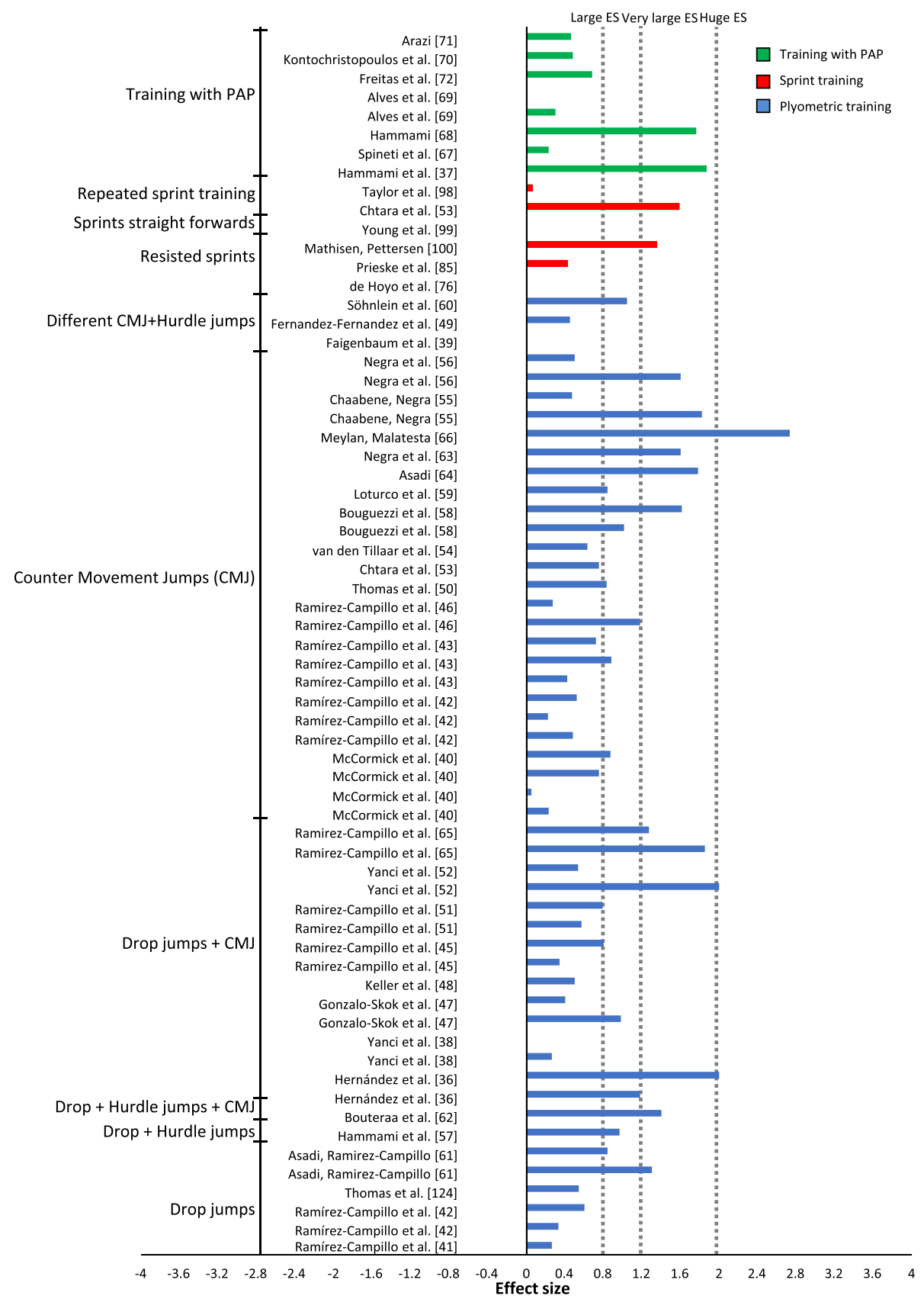

Fig. 6 Effect size after a plyometrics, sprint or PAP training intervention per training group

\section{Specific COD Training and Sprint Training}

Interventions targeting specific training of COD displayed an average change in percentage from $-2.88 \%$ [85] to $6.83 \%$ [94], with effect size varying between no ES and very large ES (Figs. 5 and 7). The intervention with the greatest improvement trained in small-sided games [94]. The training interventions lasted from 4 to 10 weeks, with one, two or three training sessions a week (Table 3). All but three experimental groups [85, 86] displayed an improvement in percentage change. Two experimental groups training in repeated COD displayed very large ES [51, 91], followed by four groups assessing small-sided games revealing very large ES [94, 95] and large ES [95, 96]. The remaining groups revealed very small ES [87, 92], small ES [88, 96] and medium ES [89-93]. The effects of specific COD training were relatively low in comparison to control groups (experimental $2.4 \%$ vs. control $1.2 \%$ ).

Experimental groups performing sprints displayed an average percentage change between $-0.38 \%$ and $5.22 \%$ 


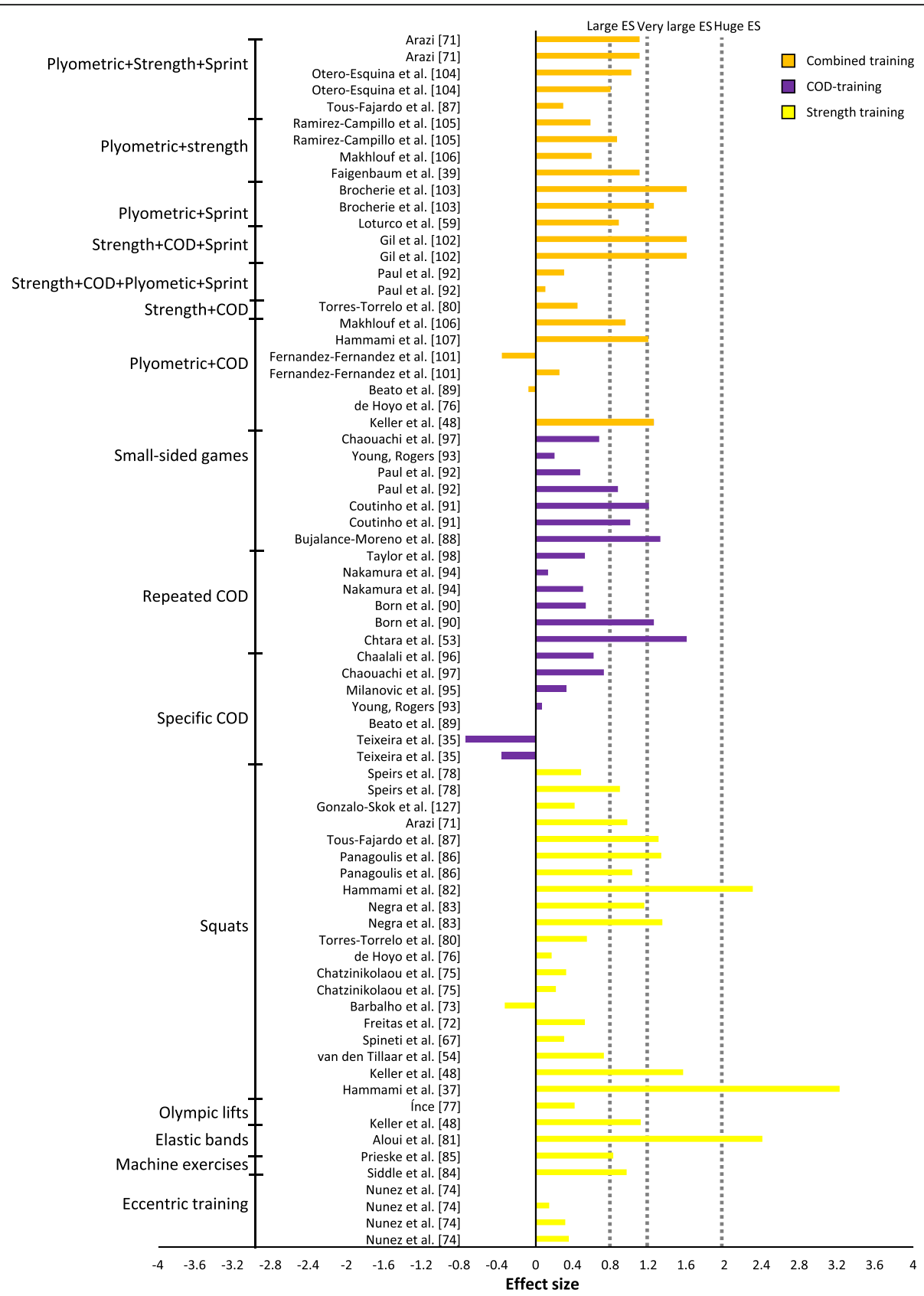

Fig. 7 Effect size after a combined, strength or COD training intervention per training group

(Table 3). Two experimental groups revealed no improvement [77, 98], followed by two groups with very small [93] and small ES [72]. In contrast, two experimental groups revealed very large ES [51, 97]. Sprint training resulted in the following average change in percentage: experimental $2 \%$ versus control $0.12 \%$.

\section{Combined Training}

A mixture of different training forms resulted in a percentage change of between $-0.66 \%$ [99] and $8.2 \%$ [69] (Table 4, Fig. 5); effects size varied from small ES to very large ES (Fig. 7). The training intervention that produced the greatest results performed plyometrics and sprint training [69]. The training interventions lasted between 5 and 8 weeks, with one to three training sessions a week. All but three experimental groups revealed an improvement post-intervention [77, 86, 99]. Furthermore, five experimental groups displayed very large ES [42, 102, 103], whereas eight groups displayed large ES [60, 69, 100, 101, 104, 105], three groups displayed medium ES [101, 104, 105] and five groups displayed small ES [78, 84, 96, 99] (Fig. 7). 
All experimental groups were formed by seven or more subjects. Eleven experimental groups assessed a strength-oriented COD test to measure performance, followed by five velocity-oriented and eight mixed COD tests. Combined training resulted in an increase in COD performance of $3.18 \%$ for the experimental group after training interventions versus $0.8 \%$ for the control groups.

\section{Discussion}

The main objectives of this review were to examine previous literature on (1) how plyometric, strength, sprint, specific COD training, training for PAP or a combination of these approaches can be performed to improve COD performance, and (2) which form of training is optimal for improvement in COD. Most of the studies revealing large ES employed plyometric training. Studies that included drop jumps and/or countermovement jumps displayed the highest percentage increase in performance (Fig. 4). Strength training resulted in the highest ES [63] (Fig. 7).

\section{Plyometric Training}

Of the experimental groups assessing plyometric training, 23 achieved ES $>0.8$ and 28 achieved ES $<0.8$ (Fig. 6). Of the 12 experimental groups revealing ES > 0.8, all groups trained twice a week, except Bouguezzi et al. [53]. Previous research on plyometric training frequency indicates that moderate training frequency (approximately two training sessions a week) is optimal in comparison to higher training frequency. de Villarreal et al. [109] suggest that moderate training frequency leads to greater athletic performance in maximal strength and $20 \mathrm{~m}$ sprints, which are physical determinants of COD performance.

An important and perhaps surprising finding with useful relevance for strength and conditioning coaches is that younger participants did not reveal greater ES than older participants. The average age of the experimental groups that displayed small ES was 15 years, followed by 15.5 years for those displaying medium ES. Experimental groups revealing ES > 1.2 were 16.1 years old on average. A possible explanation could be that jump exercises assessed in the studies are not properly designed or sufficient in terms of specificity and transferability to the respective COD tests. It is likely that a certain level of motor coordination is required for plyometric training to have a reasonable transfer to COD performance, because plyometric training can be technically challenging for younger athletes [13]. Older athletes commonly possess a greater level of strength [110]. It must also be considered that younger athletes gain greater strength after puberty because of more muscle mass [111], increased level of testosterone [112] and increased motor control
[113, 114]. Previous research points out that physically stronger athletes will benefit more from plyometric training compared to weaker athletes [13]. It is also known that females have less muscle mass and more fat mass and thereby less relative strength compared to males [110], a possible handicap when utilizing plyometric training.

One of the 16 experimental groups revealing ES below medium was composed of females with an average age of 15.7 years. Since six groups of females trained in plyometrics, it would be expected that more groups of females would achieve low ES. The results are more surprising since four experimental groups revealed large ES, as opposed to 19 for males. The experimental groups of females who displayed large ES were 19.2 years old on average versus 15.4 years for males. Therefore, the surprising COD results between sexes could be linked to the differences in biological age, although this was not investigated. Alternatively, increased muscle-tendon stiffness following plyometric training could explain some of the findings. Tendon stiffness is an important factor for performance improvement in actions that include SSC [115]. To our knowledge, it is unclear how adaptations in muscle-tendon vary by sex following plyometric training. Theoretically, the effects of females' increased COD performance could be due to similar tendon stiffness adaptations between sexes, in contrast to males' advantage in gaining muscle mass.

In all COD tests where the experimental groups revealed ES $>0.8$, the test was between 10 and $40 \mathrm{~m}$, except for one group [49]. The duration and intensity of the test will determine the release of metabolic energy during COD. While in the end, high-energy phosphates are the main source of energy during CODs, with increasing duration (several CODs over a longer period of time) the predominant pathway for energy will change [116]. Within the groups that displayed small and medium ES, three and four groups respectively used COD tests of over $40 \mathrm{~m}$ [34, 41, 43-45, 49, 61, 96]. These groups performed short duration plyometric exercises, but the applied COD tests lasted over $15 \mathrm{~s}$. The relatively low ES achieved in these groups can partly be explained by different demands in terms of energy release in plyometric training in comparison to the applied COD tests: longer COD tests will challenge the different energy systems to a greater extent [117]. Other factors could be technique while executing the COD test, familiarity with the COD test, neuromuscular and psychomotor statuses on test day, etc.

There were no consistent trends regarding studies addressing strength-oriented, velocity-oriented CODs or a mixture of both in terms of effect sizes. However, three experimental groups [46, 57] implementing velocityoriented CODs achieved ES > 1.2 versus one group [45] 
that used a force-oriented COD test. The remaining groups revealing ES $>1.2$ used a mixture of different COD angles. An important aspect of plyometric training is to enhance relative strength and rapid exertion of force to the ground [30]. These results suggest that plyometric training may be more effective for athletes that need to develop both velocity-oriented and strengthoriented COD performance.

All plyometric training interventions included countermovement jumps or drop jumps. The reason why some groups failed to achieve large ES can be more complex than in the previously stated arguments. Drop jumps could have been performed with suboptimal drop height or poor technique [13]. Data from Ramirez-Campillo et al. [43] revealed that soccer athletes performed better from optimal drop jump height (medium ES) versus fixed drop jump height (small ES). Furthermore, countermovement jumps could have been performed with a lack of specificity in terms of depth and not enough emphasis on fast muscle actions in eccentric and concentric work [117]. The benefit of drop jumps in comparison to countermovement jumps is that the eccentric phase requires a greater amount of force to be exerted in the lower extremities. Furthermore, eccentric hamstring strength, among other factors, has proved to be important during deceleration in COD [20]. Data from this review show that drop jumps along with countermovement jumps seems to be an optimal approach compared to training that involves a single exercise approach. Brughelli et al. [117] suggest that plyometric training aimed at developing COD ability should be performed both bilaterally and unilaterally, whereas force exerted to the ground is completed in vertical, horizontal and lateral directions.

\section{Training for PAP}

Seven studies formed by eight experimental groups used PAP to develop COD performance. Only two studies $[64,69]$ implemented an exercise that included a COD in the training protocol. Spineti et al. [64] involved zigzag sprints after 5-RM high pulls, the experimental group experienced a decrease in COD performance. Olympic lifts are unarguably a technically challenging exercise: if the technique is not highly developed, it could hypothetically restrict the activation of highthreshold muscle fibres and counteract the PAP effect upon the following exercises. Arazi [69] also included zig-zag sprint as part of the program and displayed small ES in untrained individuals. The lack of improvements for studies including PAP could also be explained by relatively high training frequency. Alves et al. [66] had two experimental groups performing the same training protocol, but they differed in training frequency. The group training twice a week experienced no ES and the group training once a week experienced small ES in
COD improvement. While the differences between the groups were minimal, the results suggest that higher training frequency $(\geq 2)$ may not be suitable when training for PAP. The experimental groups included several sets of plyometric training, and previous research suggests that moderate training frequency is optimal [109]. It is also very well known that strength training with repetitions close to 1RM causes substantial neuromuscular fatigue. The results are therefore unsurprising.

The lack of improvement could also be explained by the fact that there is no clear consensus regarding rest time in relation to PAP effect, in addition to the individual muscle fibre compositions, which can determine the PAP effect [118].

The remaining groups that achieved marked improvements in COD performance used half squats before plyometric exercises. The half squat is performed with a shorter range of motion, compared to the exercises used in those studies with poor results. Previous research [119] reveals that squats performed with a shortened range of motion improve performance in jumps and sprints in comparison to deeper squats. Two of the groups revealing great improvement used the $T$ test with $90^{\circ}$ and $180^{\circ}$ direction changes. Considering that force- and velocityoriented CODs are determined by both COD angle and approaching velocity [13], the $180^{\circ}$ direction change in the $T$ test is arguably velocity-oriented, due to sideways running prior to the COD which causes a relatively low velocity when entering the $180^{\circ}$ angle of direction change. Less vertical displacement of COM is expected in velocity-oriented CODs, which means a shorter range of motion, as in half squats. Theoretically, and based on these findings, the effect of studies implementing half squats could be threefold:

1) Relatively high number of reps in squat sets could have an isolated effect on the subject's strength gain, thus making plyometric training more effective in the subsequent training session.

2) Considering that an increase in neural drive occurs at the specific angles trained [120], it is reasonable that the acute PAP effect is joint angle-specific, giving half squats an advantage over deep squats at generating increased neural drive for the exercises performed with similar joint angles within the same training session.

3) If half squats, in fact, encompass similar joint angles as in the COD test, new strength gain from the exercise is likely to transfer to the applied COD test post-intervention due to the principle of specificity as introduced by Henry [121].

A trend in PAP training is the lack of specific exercises: only one study [64] included force exertion in a 
lateral direction, braking or reacceleration in the training programme. No clear conclusion can be drawn on the effect of this form of training upon COD performance because of the low number of studies. Future research should expand the knowledge regarding PAP training in relation to COD performance in general, and how exercises performed at specific joint angles influence the PAP effect.

\section{Strength Training}

Of 29 experimental groups, 14 displayed ES $>0.8$ (average age 16.5 years), whereas three of these studies revealed huge ES (>2); all trained twice a week for 8 weeks. The tests applied to measure COD performance included at least one strength-oriented COD. Two studies revealing huge ES trained using half squats $[63,80]$ at $70-90 \%$ of $1 \mathrm{RM}$, and one study trained with elastic bands [73]. Aloui et al. [73] implemented elastic band training, which consisted of knee and hip extension exercises, where the participants progressively increased the number of sets and elastic band resistance during the training period. The group training with elastic bands were on average 18.3 years old, while both the groups training with half squats were on average 16.2 years old. The larger improvements can be explained by the role of puberty in physical development and the fact that most gains due to neurological adaptations following a maximal strength programme are achieved in the first weeks [122-124].

The remaining experimental groups displaying ES > 0.8 trained with Olympic lifts [42] and different squat variations, one study training in the Nordic hamstring exercise [71]. The study implementing the eccentric Nordic hamstring exercise increased COD performance by $2.46 \%$, supporting statements in previous research regarding the importance of eccentric hamstring strength during the deceleration phase in COD [20].

The experimental groups showing medium ES trained with squats twice per week. In contrast, the groups that had below medium ES trained with squats and other multi-joint exercises, Olympic lifts and eccentric strength exercises 2.5 times a week on average. These groups did not differ in age and the COD tests to measure performance were mostly strength-oriented for all groups. The only difference between the medium ES and $<0.5$ ES group was some variation in exercise selection and training frequency.

Monotonous training with squats can be problematic: while it demands great force exertion, no propulsion occurs as in COD. During a traditional squat, force is produced vertically, but deceleration and acceleration in COD require vertical-horizontal exertion of force [21].

These results suggest that a moderate strength training frequency seems to be optimal and squats need to be combined with motions that create horizontal exertion of force to give reasonable transfer to COD performance. Furthermore, the group displaying medium ES was three years older than the groups displaying ES > 0.8. Therefore, it is reasonable to think that differences between these groups are age-related rather than due to exercise selection and training volume.

\section{Specific COD Training}

With respect to 20 experimental groups performing specific COD training, there were six experimental groups revealing ES > 0.8 (Table 3 ). These six groups were 16.8year-old participants with 14 training sessions on average, in comparison to the remaining 14 groups that were, on average, 17.3 years old with 13 training sessions during the intervention period. In other words, the experimental groups revealing marked improvement did not differ much from those revealing less improvement in terms of total training volume and age.

In two studies [51, 91] where the experimental groups displayed very large ES, repeated COD was trained with the same distance and angle used to measure improvement in COD after the intervention. Two experimental groups [95] with ES $>0.8$ that trained in small-sided games (which include repeated COD) also conducted repeated COD tests. Taking this into account, training specificity, in addition to age, could explain the occurrence of large ES post-intervention. Younger athletes have less experience with COD training, and training specifically towards the COD test that measures improvement can be advantageous in comparison to groups that are not training specifically enough. This is exemplified by Chaalali et al. [90], who implemented specific COD training with a ball among young soccer players and measured improvement with a 505 agility test. Alongside the number and angle of CODs, the use of a ball complicates the COD task, where the focus on properly handling the ball may prevent the athletes from a rapid completion of the COD task. COD training is effective for young people if the task prevents complications [13].

The lack of specificity can be exemplified by Nakamura et al. [92]. Despite training with $18-\mathrm{m}$ repeated sprints with two CODs, they measured performance with a 70-m COD test with three CODs, resulting in very small to medium $\mathrm{ES}$ ( $\mathrm{ES}=0.13$ and 0.5 ). Both Chaouachi et al. [89] and Young and Rogers [87] used small-sided games in their interventions, showing very small to medium ES post-intervention $(\mathrm{ES}=0.67$ and 0.2 . Differences in percentage increases were also apparent, being $2.5 \%$ and $0.8 \%$ respectively. The difference between the two studies is that Chaouachi et al. [89] demonstrated medium ES by conducting several CODs with small angles of new direction when measuring COD performance. Since the experimental groups were 
composed of soccer players in both studies, the results can be explained by the fact that the execution of strength-oriented COD in small-sided games in soccer is not necessary, but more velocity-oriented COD is apparent. Arguably, a different COD test could have been used to track performance post-intervention, since the technique in a COD varies according to the angle and entry speed of the task. Additionally, the same energy systems that are utilized in competition should be targeted during COD training, as exemplified by the effect of repeated COD training upon the ability to reproduce high intensity CODs during competition [21].

\section{Sprint Training}

Six experimental groups performed sprint training, and only two groups revealed ES $>0.8$. These two groups trained once a week and were on average 14.6 years old. The remaining four groups with ES $<0.5$ were 22 years old on average, and three of the groups trained twice a week. However, the last group trained three times a week for a period of only 2 weeks. de Villarreal et al. [109] point out that small to moderate training frequency $(<2)$ during a week is most effective to develop physical abilities like sprint, jump and maximal strength. Given that experimental groups that revealed large ES were formed by younger subjects, technical learning effects could explain differences related to performance.

Training groups revealing no ES to small ES performed 20 to $60 \mathrm{~m}$ COD tests that included between three and nine CODs of $100^{\circ}$ or more respectively [72, 77, 93, 98]. During the acceleration phase of sprints, horizontal force is exerted to the ground, followed by vertical adjustment of the body at greater velocity. Bourgeois et al. [13] suggest that runs of fewer than 10-m should be assessed to develop COD ability. Furthermore, the number of changes in direction will influence the specificity regarding sprint training: several COD manoeuvres with exertion of force to the ground in different directions differentiate the muscle work performed in CODs from that in sprints. Previous work points out the need to perform actions in training that are similar to movement patterns performed in competition [21]. Differences in movement patterns are challenging when performing straight-line sprint training to develop COD performance, since the technique is different from COD, even though the acceleration phase is similar $[13,20]$. Nevertheless, few experimental groups have assessed sprint training in this context, making it difficult to determine which form of sprint training is optimal to enhance COD performance.

\section{Combined Training}

Of the 24 experimental groups with a combined approach to training for COD performance, only six groups were not composed of soccer players $[69,78,99,100]$. Gil et al.
[102], who showed very large ES in two experimental groups, implemented squat jumps plus COD and sprint training in their training protocol. The result was impressive, considering the athletes were 22 and 23-year-old professional athletes. However, it is worth noting that the athletes were in the pre-season training period. The author states that a significant amount of training, such as small-sided games, was performed in addition to the experimental training protocol. It is also worth noting that previous research in this review has revealed large effects of squat training on COD performance (Table 2). Previous research [35] has revealed that squat jumps with countermovement and an external load of $30-60 \%$ of body mass are optimal for exerting maximal power output. This is also a load used in previous studies training for PAP [125], which, theoretically, could enhance performance in CODs and sprints within and after the training session. These are factors that could explain some of the results.

All eight experimental groups revealing large ES had plyometrics in their training protocol. Six of these groups had an average age of 17 or more, whereas the two remaining groups were on average 14.5 and 11.3 years old and trained in COD in addition to plyometric training. The results in relation to the groups displaying large ES can be explained by previous findings in this review, which highlight that plyometric training seems to be beneficial for older athletes due to increased strength after puberty. However, improvements in COD ability could also be a result of little experience, thus making specific COD training a great stimulus for improvements in younger athletes. In contrast, the remaining groups, which display ES $>0.8$ and included plyometric training in their training programme were 15.6 years old on average.

The current review explored multiple approaches to training, which included variations concerning age and sex of the athletes, number of training sessions and use of different COD tests to measure performance. Thus, discovering clear trends and conclusions is difficult.

When combining training forms, it is impossible to distinguish between the isolated effects of each individual training form on COD performance. Apart from studies that included plyometric training, several studies revealed impressive results when including sprint and strength training. The benefit of performing combined training is that an athlete can improve multiple determinant factors upon which COD performance depends. In court and field sports, situations occur in which entry speed and angle of the COD task vary, which could support the function of combined training to develop COD performance. With the development of multifactorial determinants, which a COD performance depends upon, the athlete is more primed to fulfil competitive demands in a variety of match situations [21]. 


\section{Practical Consequences and Applications}

After calculations are made regarding effect sizes, there is no indication of one training form being superior to any other. All training forms contained at least two experimental groups that displayed large ES. Strength and conditioning coaches should aim to optimize training in COD with respect to sport-specific demands and individual players' role. The effect of different training forms on COD performance can be explained by the forcevelocity relationship [126]. Rapid muscle actions detach myosin heads and lead to fewer cross-bridges being activated in sarcomeres. Increased contraction velocity will, therefore, lead to a decrease in muscle force [126]. This review reveals that both strength training and plyometric training are effective training forms in sports that utilize strength-oriented COD, because strength-oriented CODs feature a longer stretch-shortening cycle and greater exertion of muscle force in comparison to velocity-oriented CODs. Sprint training should be implemented to develop velocity-oriented $C O D$ in sports where the magnitude and number of CODs are lower, and where sprints followed by COD are performed over longer distances $(>10 \mathrm{~m})$. Velocity-oriented COD is characterized by rapid muscle actions, as occur during sprinting.

Strength and conditioning coaches should be careful when employing CODs over longer distances with several changes in direction. While anaerobic energy systems play a central role in COD performance, practice of several COD manoeuvres over greater distances will target the aerobic energy system more, while making the COD task more complicated [13].

The main consideration when developing COD performance is specificity in the respective sport when organizing the length, magnitude and number of COD manoeuvres in the COD task. Field and court athletes will perform different types of COD during competition; combined training can lead to a wider spectrum of capabilities regarding COD performance. Nevertheless, COD is a situational ability, not a general ability, though increased performance in a single COD task can lead to increased performance in a different COD task [13].

\section{Conclusion}

The ability to perform COD effectively is an important skill in numerous sports, thus making it relevant to investigate which forms of training lead to improvement in this skill. The most important finding in this review is that plyometric training, strength training, sprint training, COD training and a combination of these training forms can be used to develop COD performance. The optimal form of training depends on the types of COD involved in different sports. In general, strength training is sufficient to develop strength-oriented COD, plyometric training is effective in developing both strength- and velocity-oriented $\mathrm{COD}$, and sprint training is beneficial for velocity-oriented COD. Specific COD training can be implemented, but there will be a lack of transferability unless the task is comparable with respect to running patterns in competitive situations. Finally, a combination of the mentioned training forms can be used to develop COD performance if the training is similar to COD in terms of muscle work and duration.

\section{Abbreviations \\ CG: Control group; CMJ: Countermovement jump; COD: Change of direction; COM: Centre of mass; DJ: Drop jump; Dom-leg: Dominant leg; \\ EG: Experimental group; ES: Effect size; N-Dom leg: Non-dominant leg; None: Control group not provided; PAP: Post-activation potentiation; Post: Post-test; Pre: Pre-test; R: Repetitions; RDL: Romanian deadlift; RM: Repetition maximum; RSA: Repeated sprint ability test; S: Series; SSC: Stretch-shortening cycle; Tr: Training}

\section{Acknowledgements}

Not applicable.

\section{Authors' Contributions}

All authors contributed equally to the manuscript. All authors read and approved the final manuscript.

\section{Funding}

This study was conducted without any funding from companies, manufacturers or outside organizations.

\section{Availability of Data and Materials}

Please contact author for data requests.

\section{Ethics Approval and Consent to Participate}

Not applicable

\section{Consent for Publication}

Not applicable

\section{Competing Interests}

The authors, Halvard Nygaard Falch, Håvard Guldteig Rædergård, and Roland van den Tillaar, declare that they have no competing interests relevant to the content of this review.

Received: 30 April 2019 Accepted: 31 October 2019

Published online: 19 December 2019

\section{References}

1. Stølen T, Chamari K, Castagna C, Wisløff U. Physiology of soccer. Sports Med. 2005;35(6):501-36.

2. Köklü Y, Alemdaroğlu U, Özkan A, Koz M, Ersöz G. The relationship between sprint ability, agility and vertical jump performance in young soccer players. Sci Sports. 2015;30(1):e1-5.

3. DeWeese B, Nimphius S. Program design and technique for speed and agility training. In: Essentials of strength training and conditioning. Champaign: Human Kinetics; 2016. p. 521-58.

4. Bangsbo J, Mohr M, Krustrup P. Physical and metabolic demands of training and match-play in the elite football player. J Sports Sci. 2006;24(07):665-74.

5. Bradley PS, Sheldon W, Wooster B, Olsen P, Boanas P, Krustrup P. Highintensity running in English FA Premier League soccer matches. J Sports Sci. 2009;27(2):159-68.

6. Helgerud J, Engen LC, Wisløff U, Hoff J. Aerobic endurance training improves soccer performance. Med Sci Sports Exerc. 2001;33(11):1925-31.

7. Carling C, Bloomfield J, Nelsen L, Reilly T. The role of motion analysis in elite soccer. Sports Med. 2008;38(10):839-62.

8. Bloomfield J, Polman R, O'Donoghue P. Physical demands of different positions in FA Premier League soccer. J Sports Sci Med. 2007;6(1):63-71.

9. Withers R, Maricic Z, Wasilewski S, Kelly L. Match analysis of Australian professional soccer players. J Hum Mov Stud. 1982;8(4):159-76. 
10. Póvoas SC, Seabra AF, Ascensão AA, Magalhães J, Soares JM, Rebelo AN. Physical and physiological demands of elite team handball. J Strength Cond Res. 2012;26(12):3365-75.

11. Conte D, Favero TG, Lupo C, Francioni FM, Capranica L, Tessitore A. Timemotion analysis of Italian elite women's basketball games: individual and team analyses. J Strength Cond Res. 2015;29(1):144-50.

12. Duthie GM, Pyne DB, Marsh DJ, Hooper SL. Sprint patterns in rugby union players during competition. J Strength Cond Res. 2006;20(1):208-14.

13. Bourgeois F, McGuigan M, Gill N, Gamble P. Physical characteristics and performance in change of direction tasks: A brief review and training considerations. J Austr Strength Cond. 2017;25(5):104-17.

14. Sheppard JM, Young WB. Agility literature review: classifications, training and testing. J Sports Sci. 2006;24(9):919-32.

15. Castillo-Rodríguez A, Fernández-García JC, Chinchilla-Minguet JL, Carnero EÁ. Relationship between muscular strength and sprints with changes of direction. J Strength Cond Res. 2012;26(3):725-32.

16. Jones $P$, Bampouras T, Marrin K. An investigation into the physical determinants of change of direction speed. J Sports Med Phys Fit. 2009; 49(1):97-104.

17. Young W, James R, Montgomery I. Is muscle power related to running speed with changes of direction? J Sports Med Phys Fit. 2002;42(3):282-8.

18. Peterson MD, Alvar BA, Rhea MR. The contribution of maximal force production to explosive movement among young collegiate athletes. J Strength Cond Res. 2006;20(4):867-73.

19. Chaouachi A, Brughelli M, Chamari K, Levin GT, Abdelkrim NB, Laurencelle L, et al. Lower limb maximal dynamic strength and agility determinants in elite basketball players. J Strength Cond Res. 2009;23(5):1570-7.

20. Chaouachi A, Manzi V, Chaalali A, Wong DP, Chamari K, Castagna C. Determinants analysis of change-of-direction ability in elite soccer players. J Strength Cond Res. 2012;26(10):2667-76.

21. Young W, Farrow D. A review of agility: practical applications for strength and conditioning. Strength Cond J. 2006;28(5):24-9.

22. Potach D, Chu D. Program design and technique for plyometric training. In: NSCA, editor. Essentials of strength training and conditioning. Human Kinetics; 2016. p. 471-520

23. Lockie RG, Schultz AB, Callaghan SJ, Jeffriess MD, Luczo TM. Contribution of leg power to multidirectional speed in field sport athletes. J Austr Strength Cond. 2014:22(2):16-24.

24. Vescovi JD, Mcguigan MR. Relationships between sprinting, agility, and jump ability in female athletes. J Sports Sci. 2008;26(1):97-107.

25. Condello G, Minganti C, Lupo C, Benvenuti C, Pacini D, Tessitore A. Evaluation of change-of-direction movements in young rugby players. Int J Sports Physiol Perf. 2013;8(1):52-6.

26. Gabbett TJ, Kelly JN, Sheppard JM. Speed, change of direction speed, and reactive agility of rugby league players. J Strength Cond Res. 2008;22(1):174-81.

27. Jakovljevic ST, Karalejic MS, Pajic ZB, Macura MM, Erculj FF. Speed and agility of 12-and 14-year-old elite male basketball players. J Strength Cond Res. 2012;26(9):2453-9.

28. Hewit JK, Cronin JB, Hume PA. Kinematic factors affecting fast and slow straight and change-of-direction acceleration times. J Strength Cond Res. 2013;27(1):69-75.

29. Markovic G. Poor relationship between strength and power qualities and agility performance. J Sports Med Phys Fit. 2007:47(3):276-83.

30. Nimphius S, Mcguigan MR, Newton RU. Relationship between strength, power, speed, and change of direction performance of female softball players. J Strength Cond Res. 2010;24(4):885-95.

31. Spiteri T, Nimphius S, Hart NH, Specos C, Sheppard JM, Newton RU. Contribution of strength characteristics to change of direction and agility performance in female basketball athletes. J Strength Cond Res. 2014;28(9):2415-23.

32. Bishop C, Turner AN, Cree J, Maloney S, Marshall J, Jarvis P. Postactivation potentiation and change of direction speed in elite academy rugby players. J Strength Cond Res. 2017(published ahead of Print). https://doi.org/10. 1519/JSC.0000000000001834.

33. Ramírez-Campillo R, Meylan C, Álvarez C, Henríquez-Olguín C, Martínez C, Cañas-Jamett $R$, et al. Effects of in-season low-volume high-intensity plyometric training on explosive actions and endurance of young soccer players. J Strength Cond Res. 2014;28(5):1335-42.

34. Ramírez-Campillo R, Burgos CH, Henríquez-Olguín C, Andrade DC, Martínez C, Álvarez C, et al. Effect of unilateral, bilateral, and combined plyometric training on explosive and endurance performance of young soccer players. J Strength Cond Res. 2015;29(5):1317-28.
35. Thomas GA, Kraemer WJ, Spiering BA, Volek JS. Maximal power at different percentages of one repetition maximum: influence of resistance and gender. J Strength Cond Res. 2007;21(2):336-42.

36. Asadi A, Ramirez-Campillo R. Effects of cluster vs. traditional plyometric training sets on maximal-intensity exercise performance. Medicina. 2016; 52(1):41-5. https://doi.org/10.1016/j.medici.2016.01.001.

37. Hammami M, Negra Y, Aouadi R, Shephard RJ, Chelly MS. Effects of an inseason plyometric training program on repeated change of direction and sprint performance in the junior soccer player. J Strength Cond Res. 2016; 30(12):3312-20

38. Bouteraa I, Negra Y, Shephard RJ, Chelly MS. Effects of combined balance and plyometric training on athletic performance in female basketball players. J Strength Cond Res. 2018(published ahead of print). https://doi. org/10.1519/JSC.0000000000002546.

39. Hernández S, Ramirez-Campillo R, Álvarez C, Sanchez-Sanchez J, Moran J, Pereira LA, et al. Effects of plyometric training on neuromuscular performance in youth basketball players: a pilot study on the influence of drill randomization. J Sports Sci Med. 2018;17(3):372-8.

40. Yanci J, Los Arcos A, Camara J, Castillo D, García A, Castagna C. Effects of horizontal plyometric training volume on soccer players' performance. Res Sports Med. 2016;24(4):308-19.

41. Gonzalo-Skok O, Sánchez-Sabaté J, Izquierdo-Lupón L. Sáez de Villarreal E. Influence of force-vector and force application plyometric training in young elite basketball players. Eur J Sports Sci. 2018;19(3):305-14.

42. Keller S, Koob A, Corak D, Born D. How to improve change-of-direction speed in junior team sport athletes-horizontal, vertical, maximal, or explosive strength training? J Strength Cond Res. 2018. https://doi.org/10. 1519/JSC.0000000000002814.

43. Ramirez-Campillo R, Alvarez C, García-Pinillos F, Sanchez-Sanchez J, Yanci J, Castillo D, et al. Optimal reactive strength index: is it an accurate variable to optimize plyometric training effects on measures of physical fitness in young soccer players? J Strength Cond Res. 2018;32(4):885-93.

44. Ramirez-Campillo R, Álvarez C, García-Pinillos F, García-Ramos A, Loturco I, Chaabene $\mathrm{H}$ et al. Effects of combined surfaces vs. single-surface plyometric training on soccer players' physical fitness. J Strength Cond Res. 2019. https://doi.org/10.1519/JSC.0000000000002929.

45. Yanci J, Castillo D, Iturricastillo A, Ayarra R, Nakamura FY. Effects of two different volume-equated weekly distributed short-term plyometric training programs on futsal players' physical performance. J Strength Cond Res. 2017;31(7):1787-94.

46. Ramirez-Campillo R, Garcia-Pinillos F, Garcia-Ramos A, Yanci J, Gentil P, Chaabene $\mathrm{H}$, et al. Effects of different plyometric training frequencies on components of physical fitness in amateur female soccer players. Front Physiol. 2018;9:934. https://doi.org/10.3389/fphys.2018.00934.

47. McCormick BT, Hannon JC, Newton M, Shultz B, Detling N, Young WB. The effects of frontal-and sagittal-plane plyometrics on change-of-direction speed and power in adolescent female basketball players. Int J Sports Physiol Perf. 2016;11(1):102-7.

48. Ramírez-Campillo R, Gallardo F, Henriquez-Olguín C, Meylan CM, Martínez C, Álvarez C, et al. Effect of vertical, horizontal, and combined plyometric training on explosive, balance, and endurance performance of young soccer players. J Strength Cond Res. 2015;29(7):1784-95.

49. Ramirez-Campillo R, Alvarez C, Gentil P, Loturco I, Sanchez-Sanchez J Izquierdo $M$ et al. Sequencing effects of plyometric training applied before or after regular soccer training on measures of physical fitness in young players. J Strength Cond Res. 2018(published ahead of print). https://doi. org/10.1519/JSC.0000000000002525.

50. Thomas K, French D, Hayes PR. The effect of two plyometric training techniques on muscular power and agility in youth soccer players. J Strength Cond Res. 2009;23(1):332-5.

51. Chtara M, Rouissi M, Haddad M, Chtara H, Chaalali A, Owen A, et al. Specific physical trainability in elite young soccer players: efficiency over 6 weeks' inseason training. Biol Sport. 2017;34(2):137-48.

52. van den Tillaar R, Waade $L$, Roaas $T$. Comparison of the effects of 6 weeks of squat training with a plyometric training programme upon different physical performance tests in adolescent team handball players. Acta Kinesiol Uni Tart. 2015;21:75-88.

53. Bouguezzi $R$, Chaabene $H$, Negra $Y$, Ramirez-Campillo $R$, Jlalia $Z$, Mkaouer $B$ et al. Effects of different plyometric training frequency on measures of athletic performance in prepuberal male soccer players. J Strength Cond Res. 2018(Published ahead of print). https://doi.org/10.1519/JSC.0000000000002486. 
54. Loturco I, Kobal R, Kitamura K, Cal Abad CC, Faust B, Almeida L, et al. Mixed training methods: effects of combining resisted sprints or plyometrics with optimum power loads on sprint and agility performance in professional soccer players. Front Physiol. 2017;8:1034.

55. Asadi A. Effects of in-season short-term plyometric training on jumping and agility performance of basketball players. Sport Sci Health. 2013; 9(3):133-7.

56. Negra Y, Chaabene H, Fernandez-Fernandez J, Sammoud S, Bouguezzi R, Prieske $\mathrm{O}$, et al. Short-term plyometric jump training improves repeatedsprint ability in prepuberal male soccer players. J Strength Cond Res. 2018. https://doi.org/10.1519/JCS.0000000000002703.

57. Meylan C, Malatesta D. Effects of in-season plyometric training within soccer practice on explosive actions of young players. J Strength Cond Res. 2009; 23(9):2605-13

58. Chaabene $H$, Negra $Y$. The Effect of plyometric training volume on athletic performance in prepubertal male soccer players Helmi Chaabene and Yassine Negra. Int J Sports Physiol Perf. 2017;12(9):1205-11.

59. Negra Y, Chaabene H, Sammoud S, Bouguezzi R, Mkaouer B, Hachana Y, et al. Effects of plyometric training on components of physical fitness in prepuberal male soccer athletes: The role of surface instability. J Strength Cond Res. 2017:31(12):3295-304.

60. Faigenbaum AD, McFarland JE, Keiper FB, Tevlin W, Ratamess NA, Kang J, et al. Effects of a short-term plyometric and resistance training program on fitness performance in boys age 12 to 15 years. J Sports Sci Med. 2007;6(4):519-25.

61. Fernandez-Fernandez J, De Villarreal ES, Sanz-Rivas D, Moya M. The effects of 8-week plyometric training on physical performance in young tennis players. Pediat Exerc Sci. 2016;28(1):77-86.

62. Söhnlein Q, Müller $E$, Stöggl TL. The effect of 16-week plyometric training on explosive actions in early to mid-puberty elite soccer players. J Strength Cond Res. 2014;28(8):2105-14.

63. Hammami M, Negra Y, Shephard RJ, Chelly MS. The effect of standard strength vs. contrast strength training on the development of sprint, agility, repeated change of direction, and jump in junior male soccer players. $J$ Strength Cond Res. 2017;31(4):901-12.

64. Spineti J, Fiqueiredo T, Willardson J, de Oliveira BV, Assis M, de Oliveira FL, et al. Comparison between traditional strength training and complex contrast training on soccer players. J Sports Med Phys Fit. 2019;59(1):42-9.

65. Hammami M, Gaamouri N, Aloui G, Shephard RJ, Chelly MS. Effects of a complex strength-training program on athletic performance of junior female handball players. Int J Sports Physiol Perf. 2019;14(2):163-9.

66. Alves JMVM, Rebelo AN, Abrantes C, Sampaio J. Short-term effects of complex and contrast training in soccer players' vertical jump, sprint, and agility abilities. J Strength Cond Res. 2010;24(4):936-41.

67. Freitas T, Calleja-González J, Carlos-Vivas J, Marín-Cascales E, Alcaraz PE. Short-term optimal load training vs a modified complex training in semiprofessional basketball players. J Sports Sci. 2019;37(4):434-42.

68. Kontochristopoulos N, Bogdanis GC, Paradisis G, Tsolakis C. Effect of a supplementary periodized complex strength training and tapering period on postactivation potentiation of sport-specific explosive performance in adolescent national-level fencers. J Strength Cond Res. 2019. https://doi.org/ 10.1519/jsc.0000000000002967.

69. Arazi H, Asadi A, Roohi S. Enhancing muscular performance in women: compound versus complex, traditional resistance and plyometric training alone. J Muscl Res. 2014;17(2):1450007

70. Núñez FJ, Santalla A, Carrasquila I, Asian JA, Reina Jl, Suarez-Arrones LJ. The effects of unilateral and bilateral eccentric overload training on hypertrophy, muscle power and COD performance, and its determinants, in team sport players. PloS one. 2018:13(3):e0193841.

71. Siddle J, Greig M, Weaver K, Page RM, Harper D, Brogden CM. Acute adaptations and subsequent preservation of strength and speed measures following a Nordic hamstring curl intervention: a randomised controlled trial. J Sports Sci. 2019;37(8):911-20. https://doi.org/10.1080/02640414.2018.1535786.

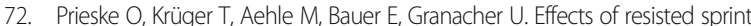
training and traditional power training on sprint, jump, and balance performance in healthy young adults: a randomized controlled trial. Front Physiol. 2018;9:156.

73. Aloui G, Hammami M, Fathloun M, Hermassi S, Gaamouri N, Shephard RJ, et al. Effects of an 8-week in-season elastic band training program on explosive muscle performance, change of direction, and repeated changes of direction in the lower limbs of junior male handball players. J Strength Cond Res. 2018. https://doi.org/10.1519/JSC.0000000000002786.
74. Ince I. Effects of split style olympic weightlifting training on leg stiffness vertical jump change of direction and sprint in collegiate volleyball players. Univer J Educ Res. 2019;7(1):24-31.

75. Barbalho M, Gentil P, Raiol R, Del Vecchio F, Ramirez-Campillo R, Coswig $V$. Non-linear resistance training program induced power and strength but not linear sprint velocity and agility gains in young soccer players. Sports. 2018;6(2):43

76. Chatzinikolaou A, Michaloglou K, Avloniti A, Leontsini D, Deli CK, Vlachopoulos D, et al. The trainability of adolescent soccer players to brief periodized complex training. Int J Sports Physiol Perf. 2018;13(5):645-55.

77. de Hoyo M, Gonzalo-Skok O, Sañudo B, Carrascal C, Plaza-Armas JR, Camacho-Candil F, et al. Comparative effects of in-season full-back squat, resisted sprint training, and plyometric training on explosive performance in U-19 elite soccer players. J Strength Cond Res. 2016;30(2):368-77.

78. Torres-Torrelo J, Rodríguez-Rosell D, González-Badillo JJ. Light-load maximal lifting velocity full squat training program improves important physical and skill characteristics in futsal players. J Sports Sci. 2017:35(10):967-75.

79. Negra Y, Chaabene H, Hammami M, Hachana Y, Granacher U. Effects of high-velocity resistance training on athletic performance in prepuberal male soccer athletes. J Strength Cond Res. 2016;30(12):3290-7.

80. Hammami M, Negra Y, Billaut F, Hermassi S, Shephard RJ, Chelly MS. Effects of lower-limb strength training on agility, repeated sprinting with changes of direction, leg peak power, and neuromuscular adaptations of soccer players. J Strength Cond Res. 2018;32(1):37-47.

81. Panagoulis C, Chatzinikolaou A, Avloniti A, Leontsini D, Deli CK, Draganidis $D$, et al. In-season integrative neuromuscular strength training improves performance of early-adolescent soccer athletes. J Strength Cond Res. 2018. https://doi.org/10.1519/JSC.0000000000002938.

82. Gonzalo-Skok O, Tous-Fajardo J, Valero-Campo C, Berzosa C, Bataller AV, Arjol-Serrano JL, et al. Eccentric-overload training in team-sport functional performance: constant bilateral vertical versus variable unilateral multidirectional movements. In J Sports Physiol Perf. 2017;12(7):951-8. https://doi.org/10.1123/ijspp.2016-0251.

83. Speirs DE, Bennett MA, Finn CV, Turner AP. Unilateral vs. bilateral squat training for strength, sprints, and agility in academy rugby players. J Strength Cond Res. 2016:30(2):386-92. https://doi.org/10.1519/jsc. 0000000000001096

84. Tous-Fajardo J, Gonzalo-Skok O, Arjol-Serrano JL, Tesch P. Enhancing change-of-direction speed in soccer players by functional inertial eccentric overload and vibration training. Int J Sports Physiol Perf. 2016; $11(1): 66-73$.

85. Teixeira A, Arins F, De Lucas R, Carminatti L, Dittrich N, Nakamura F, et al. Shuttle-run interval training with more directional changes induces superior gains in shuttle sprint performance in female professional futsal players. Hum Mov. 2018;19(5):40-51.

86. Beato M, Bianchi M, Coratella G, Merlini M, Drust B. Effects of plyometric and directional training on speed and jump performance in elite youth soccer players. J Strength Cond Res. 2018;32(2):289-96.

87. Young W, Rogers N. Effects of small-sided game and change-of-direction training on reactive agility and change-of-direction speed. J Sports Sci. 2014;32(4):307-14

88. Milanovic Z, Sporis G, Trajkovic N, James N, Samija K. Effects of a 12 week SAQ training programme on agility with and without the ball among young soccer players. J Sports Sci Med. 2013;12(1):97-104.

89. Chaouachi A, Chtara M, Hammami R, Chtara H, Turki O, Castagna C. Multidirectional sprints and small-sided games training effect on agility and change of direction abilities in youth soccer. J Strength Cond Res. 2014;28(11):3121-7.

90. Chaalali A, Rouissi M, Chtara M, Owen A, Bragazzi N, Moalla W, et al. Agility training in young elite soccer players: promising results compared to change of direction drills. Biol Sport. 2016;33(4):345-51.

91. Born D-P, Zinner C, Düking P, Sperlich B. Multi-directional sprint training improves change-of-direction speed and reactive agility in young highly trained soccer players. J Sports Sci Med. 2016;15(2):314-9.

92. Nakamura FY, Sanchez-Sanchez J, Ramirez-Campillo R, Petisco C, GonzaloSkok O, Rodriguez-Fernandez A et al. Effects of repeated-sprints with changes of direction on youth soccer player's performance: Impact of initial fitness level. J Strength Cond Res. 2017(published ahead of print). https:// doi.org/10.1519/JSC.0000000000002232.

93. Taylor JM, Macpherson TW, McLaren SJ, Spears I, Weston M. Two weeks of repeated-sprint training in soccer: To turn or not to turn? Int J Sports Physiol Perf. 2016;11(8):998-1004. 
94. Bujalance-Moreno P, García-Pinillos F, Latorre-Román P. Effects of a smallsided game-based training program on repeated sprint and change of direction abilities in recreationally-trained soccer players. J Sports Med Phys Fit. 2018:58(7-8):1021-8.

95. Coutinho D, Santos S, Gonçalves B, Travassos B, Wong DP, Schöllhorn W, et al. The effects of an enrichment training program for youth football attackers. PloS one. 2018;13(6):e0199008.

96. Paul DJ, Marques JB, Nassis GP. The effect of a concentrated period of soccer specific fitness training with small-sided games on physical fitness in youth players. J Sports Med Phys Fit. 2018(published ahead of print). https:// doi.org/10.23736/S0022-4707.18.08547-X.

97. Mathisen E, Pettersen A. The effect of speed training on sprint and agility performance in female youth soccer players. J Phys Educ Sport. 2015;15(3):395-9.

98. Young $W$, McDowell M, Scarlett B. Specificity of sprint and agility training methods. J Strength Cond Res. 2001:15(3):315-9.

99. Fernandez-Fernandez J, Granacher U, Sanz-Rivas D, Marín JMS, HernandezDavo JL, Moya M. Sequencing effects of neuromuscular training on physical fitness in youth elite tennis players. J Strength Cond Res. 2018;32(3):849-56.

100. Hammami M, Gaamouri N, Aloui G, Shephard RJ, Chelly MS. Effects of combined plyometric and short sprint with change-of-direction training on athletic performance of male U15 handball players. J Strength Cond Res. 2019;33(3):662-75.

101. Makhlouf I, Chaouachi A, Chaouachi M, Othman AB, Granacher U, Behm DG. Combination of agility and plyometric training provides similar training benefits as combined balance and plyometric training in young soccer players. Front Physiol. 2018;9(1611):1-17.

102. Gil S, Barroso R, Crivoi do Carmo E, Loturco I, Kobal R, Tricoli V, et al. Effects of resisted sprint training on sprinting ability and change of direction speed in professional soccer players. J Sport Sci. 2018;36(17):1923-9.

103. Brocherie F, Girard O, Faiss R, Millet GP. High-intensity intermittent training in hypoxia: a double-blinded, placebo-controlled field study in youth football players. J Strength Cond Res. 2015;29(1):226-37. https://doi.org/10. 1519/jsc.0000000000000590.

104. Ramirez-Campillo R, Sanchez-Sanchez J, Gonzalo-Skok O, RodríguezFernandez A, Carretero M, Nakamura FY. Specific changes in young soccer player's fitness after traditional bilateral vs. unilateral combined strength and plyometric training. Front Physiol. 2018;9:265.

105. Otero-Esquina C, de Hoyo LM, Gonzalo-Skok Ó, Domínquez-Cobo S, Sánchez $\mathrm{H}$. Is strength-training frequency a key factor to develop performance adaptations in young elite soccer players? Eur J Sports Sci. 2017;17(10):1241-51

106. Sawilowsky SS. New effect size rules of thumb. J Mod Appl Stat Meth. 2009; 8(2):597-9.

107. Cohen J. Statistical power analysis for behavioral sciences. 2nd ed. Hillsdale: Erlbaum Associates; 1988.

108. Ramírez-Campillo R, Vergara-Pedreros M, Henríquez-Olguín C, MartínezSalazar C, Alvarez C, Nakamura FY, et al. Effects of plyometric training on maximal-intensity exercise and endurance in male and female soccer players. J Sports Sci. 2016;34(8):687-93.

109. de Villarreal ESS, González-Badillo JJ, Izquierdo M. Low and moderate plyometric training frequency produces greater jumping and sprinting gains compared with high frequency. J Strength Cond Res. 2008;22(3):715-25.

110. Miller AEJ, MacDougall J, Tarnopolsky M, Sale D. Gender differences in strength and muscle fiber characteristics. Eur J Appl Physiol Occup Physiol. 1993;66(3):254-62.

111. Seger JY, Thorstensson A. Muscle strength and electromyogram in boys and girls followed through puberty. Eur J Sports Sci. 2000;81(1-2):54-61.

112. Round JM, Jones DA, Honour JW, Nevill AM. Hormonal factors in the development of differences in strength between boys and girls during adolescence: a longitudinal study. Annals Hum Biol. 1999:26(1):49-62.

113. Dorfberger $\mathrm{S}$, Adi-Japha $\mathrm{E}$, Karni A. Sex differences in motor performance and motor learning in children and adolescents: an increasing male advantage in motor learning and consolidation phase gains. Behav brain Res. 2009;198(1):165-71. https://doi.org/10.1016/j.bbr.2008.10.033.

114. Yue G, Cole KJ. Strength increases from the motor program: comparison of training with maximal voluntary and imagined muscle contractions. J Neurophysiol. 1992;67(5):1114-23. https://doi.org/10.1152/jn.1992.67.5.1114.

115. Markovic G, Mikulic P. Neuro-musculoskeletal and performance adaptations to lower-extremity plyometric training. Sports Med. 2010;40(10):859-95.

116. Gastin PB. Energy system interaction and relative contribution during maximal exercise. Sports Med. 2001;31(10):725-41.
117. Brughelli M, Cronin J, Levin G, Chaouachi A. Understanding change of direction ability in sport. Sports Med. 2008;38(12):1045-63.

118. Tillin NA, Bishop D. Factors modulating post-activation potentiation and its effect on performance of subsequent explosive activities. Sports Med. 2009; 39(2):147-66.

119. Rhea MR, Kenn JG, Peterson MD, Massey D, Simão R, Marin PJ, et al. Jointangle specific strength adaptations influence improvements in power in highly trained athletes. Hum Mov. 2016;17(1):43-9.

120. Weir JP, Housh TJ, Weir LL, Johnson GO. Effects of unilateral isometric strength training on joint angle specificity and cross-training. Eur J Appl Physiol Occup Physiol. 1995;70(4):337-43.

121. Oxendine JB. Generality and specificity in the learning of fine and gross motor skills. Res Q. 1967;38(1):86-94.

122. Aagaard P, Simonsen E, Andersen J, Magnusson P, Dyhre-Poulsen P. Neural adaptation to resistance training: changes in evoked $\mathrm{V}$-wave and $\mathrm{H}$-reflex responses. J Appl Physiol. 2002;92:2309-18.

123. Häkkinen K, Newton RU, Gordon SE, McCormick M, Volek JS, Nindl BC, et al. Changes in muscle morphology, electromyographic activity, and force production characteristics during progressive strength training in young and older men. J Geron Series A Biol Sci Med Sci. 1998;53(6):B415-B23.

124. Tillin NA, Pain MT, Folland JP. Short-term unilateral resistance training affects the agonist-antagonist but not the force-agonist activation relationship. Muscle Nerve. 2011;43(3):375-84

125. Ali K, Ejaz Hussain M, Verma S, Ahmad I, Singla D. Complex training: an update. J Athl Enhanc. 2017;6(3):1-5.

126. Hill AV. The heat of shortening and the dynamic constants of muscle. Proc of the Royal Soci of London Series B-Biol Sci. 1938;126(843):136-95.

\section{Publisher's Note}

Springer Nature remains neutral with regard to jurisdictional claims in published maps and institutional affiliations.

\section{Submit your manuscript to a SpringerOpen ${ }^{\circ}$ journal and benefit from:}

- Convenient online submission

- Rigorous peer review

- Open access: articles freely available online

High visibility within the field

- Retaining the copyright to your article

Submit your next manuscript at $>$ springeropen.com 